AperTO - Archivio Istituzionale Open Access dell'Università di Torino

Chaos in a Nonautonomous Model for the Interactions of Prey and Predator with Effect of Water Level Fluctuation

This is a pre print version of the following article:

Original Citation:

Availability:

This version is available http://hdl.handle.net/2318/1755518

since 2020-09-17T10:18:11Z

Published version:

DOI:10.1142/S0218339020500205

Terms of use:

Open Access

Anyone can freely access the full text of works made available as "Open Access". Works made available under a Creative Commons license can be used according to the terms and conditions of said license. Use of all other works requires consent of the right holder (author or publisher) if not exempted from copyright protection by the applicable law. 


\section{Journal of Biological Systems \\ Chaos in a nonautonomous model for the interactions of prey and predator with effect of water level fluctuation. --Manuscript Draft--}

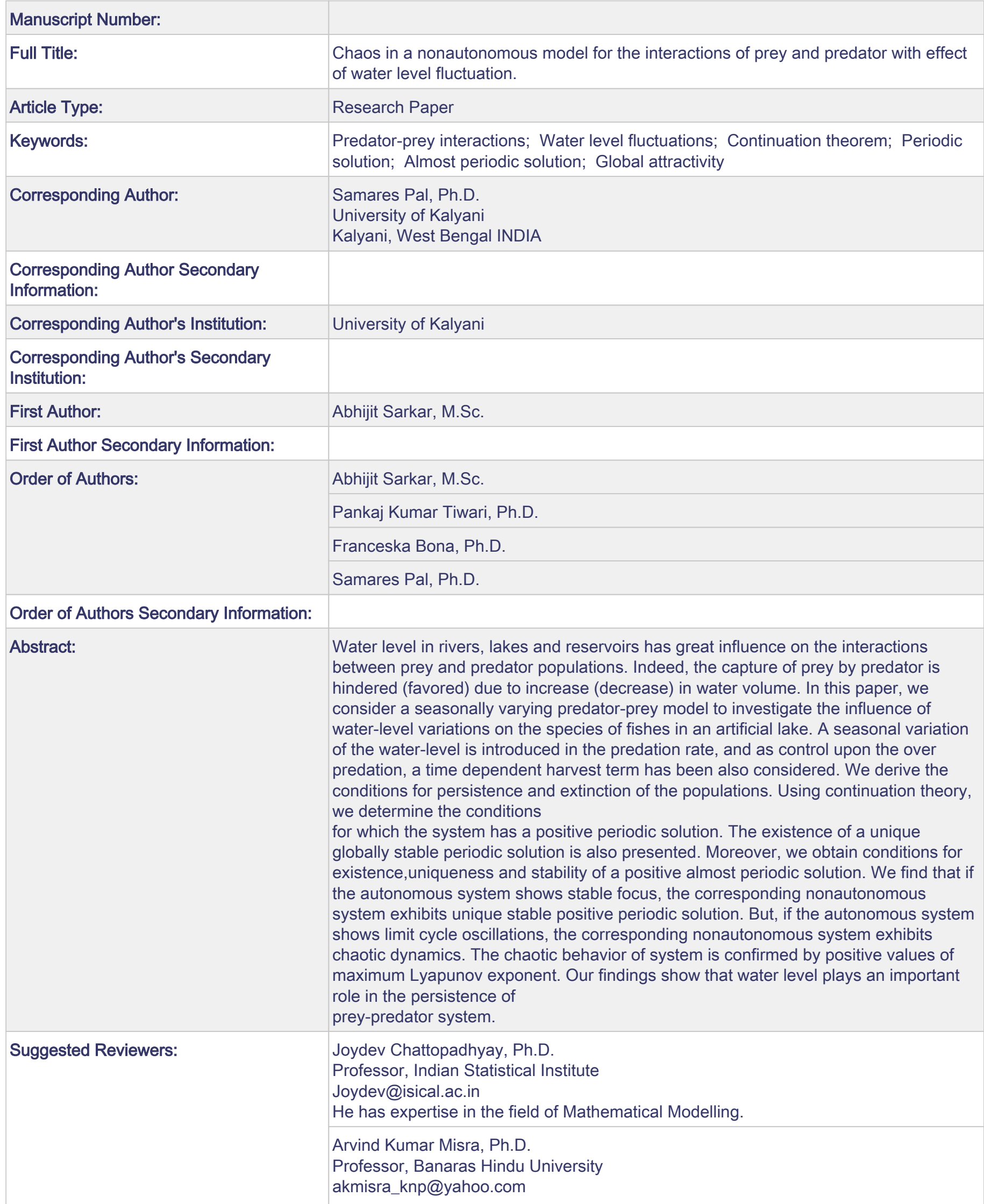


He has expertise in the field of marine plankton ecology.

Yun Kang, Ph.D.

Professor, Arizona State University

yun.Kang@asu.edu

She has expertise in the area of Nonlinear differential equations and dynamical systems.

Sudip Samanta, Ph.D.

Professor, Bankura University

samanta.sudip.09@gmail.com

He has expertise in Mathematical modelling in ecology and epidemiology with special emphasis on marine ecology.

Subhas Khajanchi, Ph.D.

Professor, Presidency University Kolkata

subhaskhajanchi@gmail.com

$\mathrm{He}$ has expertise in the field of marine plankton ecology. 


\title{
CHAOS IN A NONAUTONOMOUS MODEL FOR THE INTERACTIONS OF PREY AND PREDATOR WITH EFFECT OF WATER LEVEL FLUCTUATION
}

\author{
ABHIJIT SARKAR \\ Department of Mathematics, University of Kalyani, Kalyani - 741235, India \\ abhijit2012sarkar@gmail.com \\ PANKAJ KUMAR TIWARI \\ Department of Mathematics, University of Kalyani, Kalyani - 741235, India \\ pankajmathematics@gmail.com \\ FRANCESCA BONA \\ DBIOS, University of Turin, via Accademia Albertina 13, 10123 Turin, Italy \\ francesca.bona@unito.it \\ SAMARES PAL* \\ Department of Mathematics, University of Kalyani, Kalyani - 741235, India \\ samaresp@gmail.com
}

\begin{abstract}
Water level in rivers, lakes and reservoirs has great influence on the interactions between prey and predator populations. Indeed, the capture of prey by predator is hindered (favored) due to increase (decrease) in water volume. In this paper, we consider a seasonally varying predator-prey model to investigate the influence of water-level variations on the species of fishes in an artificial lake. A seasonal variation of the water-level is introduced in the predation rate, and as control upon the over predation, a time dependent harvest term has been also considered. We derive the conditions for persistence and extinction of the populations. Using continuation theory, we determine the conditions for which the system has a positive periodic solution. The existence of a unique globally stable periodic solution is also presented. Moreover, we obtain conditions for existence, uniqueness and stability of a positive almost periodic solution. We find that if the autonomous system shows stable focus, the corresponding nonautonomous system exhibits unique stable positive periodic solution. But, if the autonomous system shows limit cycle oscillations, the corresponding nonautonomous system exhibits chaotic dynamics. The chaotic behavior of system is confirmed by positive values of maximum Lyapunov exponent. Our findings show that water level plays an important role in the persistence of prey-predator system.
\end{abstract}

Keywords: Predator-prey interactions, Water level fluctuations, Continuation theorem, Periodic solution, Almost periodic solution, Global attractivity.

${ }^{*}$ Corresponding author 


\section{Introduction}

Lakes, ponds and rivers of mountain, temperate and tropical regions show seasonal natural fluctuations of water level. Hydrological regimes influence primary productivity ${ }^{1,2}$ and fisheries ${ }^{3,4,5,6,7}$ due to nutrient input variations from the surrounding terrestrial ecosystems and internal accumulation/resuspension of nutrient-rich sediments ${ }^{8,9,10}$. Human activities such as dam and reservoir construction, water extraction ${ }^{3,11,12}$, and in addition climate change ${ }^{7}$ enhance amplitude, frequency and unpredictability of the natural fluctuations between dry and wet conditions, especially at the littoral zone of lakes and rivers. Changes of water level cause significant variations on littoral habitat characteristics: on physical-chemical conditions of water, on coverage of shoreline vegetation ${ }^{13}$ and in general on its complexity ${ }^{14}$, then reducing its suitability as refuge and breeding area for benthic invertebrates and fish, both preys and predators ${ }^{15,16}$.

The impact of water level fluctuations in aquatic ecosystems has been studied by several researchers $17,18,19,20,21,22,23$. Depending on the spatial and temporal extension, water level fluctuations can influence structure and dynamics of the fish communities $24,25,26$. These features lead to a change in the Lotka-Volterra model 27,28 and water level variation has been included in the system of differential equations $29,30,31$. Chiboub et al. ${ }^{32}$ using a two species model showed that the seasonal variations in water level of a river exert a strong influence on prey-predator interactions between pike and roach species. Moussaoui and collaborators ${ }^{33,34}$ investigating a complex interaction between three species of fish in a lake of Southern France, showed that only under some appropriate conditions of water level the prey-predator system is permanent. Gownaris et al. ${ }^{22}$ studied the consequences of water fluctuations on ecosystem attributes of 13 African lakes. They found that in most cases water fluctuation enhanced primary and overall lake production including fisheries production but with important consequences on species diversity.

In the present paper, we investigate a complex three prey-predator system under water level fluctuations, in which the prey feeds on invertebrates, by extending the model studied by Moussaoui and Boughima ${ }^{34}$. Our main aim is to investigate rich dynamics including chaos, which was absent in earlier works. The invertebrates serve as food for both small fish (prey) and for large fish (predators). The small fish is predated by large fish. For example, this could be the system of brown trout (Salmo trutta), juveniles and adults, introduced in a mountain reservoir, and the amphipod crustacean Gammarus pulex, and other macroinvertebrates eaten by both juveniles and adults of trout. Another example of interaction potentially influenced by water level fluctuation is that of the rudd (Scardinius erithophthalmus) predated by the pike (Esox lucius) in littoral zone of lakes and ponds of temperate climate. We consider modified Beddington type interaction between invertebrates and small fish ${ }^{35}$; the interaction between large fish and invertebrates is assumed to follow ratiodependent functional response. Moreover, we consider seasonal harvesting of the large fish ${ }^{36}$. We analyze the system for positive invariance, permanence, existence 
of periodic (almost periodic) solutions, and global attractivity of unique positive periodic (almost periodic) solution ${ }^{37,38,39,40}$.

\section{The mathematical model}

Water level may influence local community dynamics in water bodies. The increase/decrease in the level of water leads to increase/decrease in the volume of water, which affects the interactions of fishes and catching capability. We examine how seasonal variations in water level and harvesting affects the outcome of preypredator interactions. Our dynamic model is a continuous time with three states, the invertebrates, the prey (small fish) and the predator (large fish), where each species is described by the evolution of its biomass $x(t), y(t)$ and $z(t)$, respectively. Let the growth rate of invertebrates follows the logistic law with $r$ as intrinsic growth rate and $K$ as environmental carrying capacity i.e., $r x(t)\left(1-\frac{x(t)}{K}\right)$. We consider modified Beddington type interaction between invertebrates, $x$ and small fish, $y$ i.e., $\frac{F x(t) y(t)}{1+h F x(t)+w F y(t)}$. Further, we assume that the interaction between large fish and invertebrates follows ratio-dependent functional response i.e., $\frac{F_{1} x(t) z(t)}{x(t)+a z(t)}$. Moreover, we consider seasonal harvesting of the large fish with $E$ as fishing effort and $q(t)$ as the catching capability. Let $d_{1}$ and $d_{2}$ be the natural death rates of prey and predator, respectively.

When a predator attacks a prey, it has access to a certain quantity of food depending on the water level. When water level is low (high), the predator is more (less) in contact with the prey. Let $b(t)$ be the accessibility function for the prey. The functions $b(t)$ and $q(t)$ are annual periodic and continuous, that is, $b(t)$ and $q(t)$ are 1-periodic. The minimum value of $b(t)$ and $q(t)$ is reached in spring, and the maximum value of $b(t)$ and $q(t)$ is attained during autumn. Denote by $\gamma$, the maximum consumption rate of resource by predator. The predator needs a quantity $\gamma z(t)$ for his food, but he has access to a quantity $\frac{b(t) y(t) z(t)}{D+z(t)}$. If $\frac{b(t) y(t)}{D+z(t)} \geq \gamma$, the predator will be satisfied with the quantity $\gamma z(t)$ for his food. On the other hand, if $\frac{b(t) y(t)}{D+z(t)} \leq \gamma$, the predator will content himself with $\frac{b(t) y(t) z(t)}{D+z(t)}$. Consequently, the quantity of food received by the predator is

$$
\min \left(\frac{b(t) y(t)}{D+z(t)}, \gamma\right) z(t)
$$

The interplay among invertebrates, small fish and large fish is depicted in Fig. 1. 


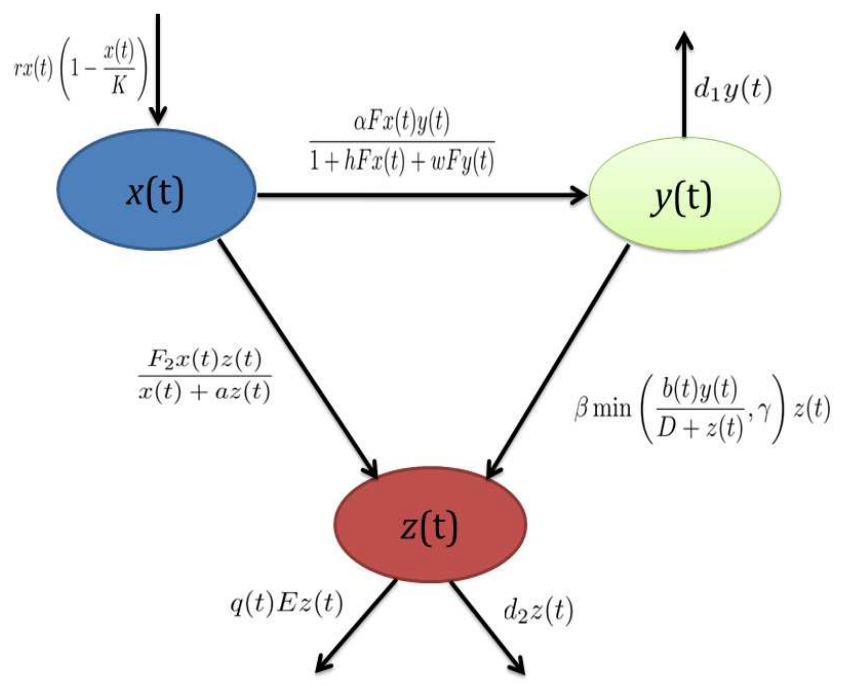

Fig. 1. Schematic diagram of the system (2.1).

Accordingly, our model is given by

$$
\begin{aligned}
\frac{d x(t)}{d t} & =r x(t)\left(1-\frac{x(t)}{K}\right)-\frac{F x(t) y(t)}{1+h F x(t)+w F y(t)}-\frac{F_{1} x(t) z(t)}{x(t)+a z(t)}, \\
\frac{d y(t)}{d t} & =\frac{\alpha F x(t) y(t)}{1+h F x(t)+w F y(t)}-\min \left(\frac{b(t) y(t)}{D+z(t)}, \gamma\right) z(t)-d_{1} y(t), \\
\frac{d z(t)}{d t} & =\beta \min \left(\frac{b(t) y(t)}{D+z(t)}, \gamma\right) z(t)+\frac{F_{2} x(t) z(t)}{x(t)+a z(t)}-d_{2} z(t)-q(t) E z(t) .
\end{aligned}
$$

The biological meaning of the parameters involved in the system (2.1) and their values used for numerical simulations are given in Table 1.

\section{Mathematical analysis and main result}

Let $x(0)=x_{0}, y(0)=y_{0}, z(0)=z_{0}$ be respectively the initial density of the invertebrates, prey and predator with $x_{0}>0, y_{0}>0$ and $z_{0}>0$. Before starting the mathematical analysis of the model (2.1), we rewrite it in a simpler form.

Proposition 3.1 For $t>0$, let $q^{l}=\min q(t), q^{u}=\max q(t), b^{l}=\min b(t)$ and 
Table 1. Biological meanings of parameters involved in the system (2.1) and their values used for numerical simulations

\begin{tabular}{ccc}
\hline Parameters & Descriptions & Values \\
\hline$r$ & Intrinsic growth rate of invertebrates in the absence of fish populations & 4.3 \\
$K$ & Carrying capacity of the environment for the invertebrates & 180 \\
$F$ & Consumption rate of invertebrate by prey & 0.8 \\
$h$ & Handling time of prey & 0.1 \\
$w$ & Prey interference coefficient & 0.1 \\
$F_{1}$ & Capture rate of prey by predator & 0.9 \\
$a$ & Half-saturation constant & 2 \\
$\alpha$ & Assimilation fraction of prey & 1.8 \\
$b$ & Accessibility function of prey & 2.15 \\
$D$ & Measure of mortality outside predation & 0.01 \\
$\gamma$ & Maximum consumption rate by predator & - \\
$d_{1}$ & Death rate of prey & 0.5 \\
$d_{2}$ & Death rate of predator & 0.45 \\
$\beta$ & Conversion rate of prey into predator & 0.3 \\
$F_{2}$ & Growth rate of predator due to invertebrates & 0.6 \\
$q$ & Catching capability & 1 \\
$E$ & Fishing effort & 0.01 \\
\hline
\end{tabular}

$b^{u}=\max b(t)$. If the following conditions hold

$$
\begin{aligned}
& d_{2}+q^{u} E<d_{1}, \\
& b^{u}<\min \left\{\frac{\gamma\left(D+z_{0}\right)}{\alpha x_{0}+y_{0}}, \frac{4 r \gamma D\left(d_{2}+q^{u} E\right)}{K \alpha\left(r+d_{2}+q^{u} E\right)^{2}}\right\},
\end{aligned}
$$

then for all $t>0$

$$
b^{u} y(t)<\gamma(D+z(t)) .
$$

Proof. Let

$$
u(t)=b^{u}(y(t)+\alpha x(t))-\gamma(D+z(t))
$$

then from condition (3.2), we have $\left.u(t)\right|_{t=0}<0$. We claim that $u(t)<0, \forall t>0$. If not, then there exists $t_{0}>0$ such that $u\left(t_{0}\right)=0$ and $\left.\frac{d u}{d t}\right|_{t_{0}} \geq 0$.

$u\left(t_{0}\right)=0 \Rightarrow b^{u}\left(y\left(t_{0}\right)+\alpha x\left(t_{0}\right)\right)-\gamma\left(D+z\left(t_{0}\right)\right)=0 \Rightarrow z\left(t_{0}\right)=\frac{b^{u}\left(y\left(t_{0}\right)+\alpha x\left(t_{0}\right)\right)}{\gamma}-D$. 
From equation (3.4), we have

$$
\begin{aligned}
\left.\frac{d u}{d t}\right|_{t_{0}} & =b^{u}\left(\frac{\alpha F x_{0} y_{0}}{1+h F x_{0}+w F y_{0}}-\frac{b\left(t_{0}\right) y_{0} z_{0}}{D+z_{0}}-d_{1} y_{0}+\alpha r x_{0}\left(1-\frac{x_{0}}{K}\right)-\frac{\alpha F x_{0} y_{0}}{1+h F x_{0}+w F y_{0}}-\frac{\alpha F_{1} x_{0} z_{0}}{x_{0}+a z_{0}}\right) \\
& \quad-\gamma\left(\beta \frac{b\left(t_{0}\right) y_{0} z_{0}}{D+z_{0}}+\frac{F_{2} x_{0} z_{0}}{x_{0}+a z_{0}}-d_{2} z_{0}-q\left(t_{0}\right) E z_{0}\right) \\
& \leq b^{u}\left(\alpha r x_{0}-\frac{\alpha r x_{0}^{2}}{K}-d_{1} y_{0}\right)+\gamma\left(d_{2} z_{0}+q\left(t_{0}\right) E z_{0}\right) \\
& \leq b^{u}\left(\alpha r x_{0}-\alpha r \frac{x_{0}^{2}}{K}-d_{1} y_{0}\right)+\gamma\left(d_{2}+q^{u} E\right)\left(\frac{b^{u}\left(y_{0}+\alpha x_{0}\right)}{\gamma}-D\right) \\
& \leq-b^{u} \frac{\alpha r x_{0}^{2}}{K}+\left\{b^{u} \alpha r+b^{u} \alpha\left(d_{2}+q^{u} E\right)\right\} x_{0}+b^{u}\left(d_{2}+q^{u} E-d_{1}\right) y_{0}-\gamma D\left(d_{2}+q^{u} E\right) \\
& \leq-\frac{b^{u} \alpha r x_{0}^{2}}{K}+b^{u}\left\{r+\left(d_{2}+q^{u} E\right)\right\} \alpha x_{0}-\gamma D\left(d_{2}+q^{u} E\right) .
\end{aligned}
$$

Right side of above inequality is quadratic polynomial whose discriminant is

$$
\Delta=b^{u 2} \alpha^{2}\left(r+d_{2}+q^{u} E\right)^{2}-\frac{4 \gamma D b_{2} \alpha r\left(d_{2}+q^{u} E\right)}{K} .
$$

From condition (3.2), it follows that $\frac{d u}{d t}<0$, which is a contradiction. So, $u(t)<$ $0, \forall t>0$.

Consequently system (2.1) is reduced to the following simple form,

$$
\begin{aligned}
\frac{d x(t)}{d t} & =r x(t)\left(1-\frac{x(t)}{K}\right)-\frac{F x(t) y(t)}{1+h F x(t)+w F y(t)}-\frac{F_{1} x(t) z(t)}{x(t)+a z(t)}, \\
\frac{d y(t)}{d t} & =\frac{\alpha F x(t) y(t)}{1+h F x(t)+w F y(t)}-\frac{b(t) y(t) z(t)}{D+z(t)}-d_{1} y(t), \\
\frac{d z(t)}{d t} & =\frac{\beta b(t) y(t) z(t)}{D+z(t)}+\frac{F_{2} x(t) z(t)}{x(t)+a z(t)}-d_{2} z(t)-q(t) E z(t) .
\end{aligned}
$$

Remark 3.1 Condition (3.2) corresponds to the scenario where interactions between predators and prey are not sufficiently strong, and thus, the accessibility function is below a threshold value given by (3.2). This situation is possible in the lake if the level of water is above a suitable value.

\section{The model with general coefficients}

First, we introduce some basic definitions and facts, which will be used throughout this paper. Let $\mathbb{R}_{+}^{3}=\left\{(x, y, z) \in \mathbb{R}^{3} \mid x \geq 0, y \geq 0, z \geq 0\right\}$. Denote by $(x, y, z)$, the solution of system (3.5) with initial condition $\left(x_{0}, y_{0}, z_{0}\right)$. For biological reasons, throughout this paper, we only consider the solutions $(x(t), y(t), z(t))$ with positive initial values, that is, $\left(x_{0}, y_{0}, z_{0}\right) \in \mathbb{R}_{+}^{3}$. Let $g(t)$ be a continuous function. If $g(t)$ is bounded on $\mathbb{R}$, we denote

$$
\bar{g}=\int_{0}^{1} g(t) d t
$$


We have the following lemma.

Lemma 4.1 Both the nonnegative and positive cones of $\mathbb{R}_{+}^{3}$ are positively invariant for system (3.5).

Proof. The solution $(x(t), y(t), z(t))$ of system (3.5) with initial values $\left(x_{0}, y_{0}, z_{0}\right)$ satisfies,

$$
\begin{aligned}
& x(t)=x_{0} \exp \left\{\int_{0}^{t}\left[r\left(1-\frac{x(s)}{K}\right)-\frac{F y(s)}{1+h F x(s)+w F y(s)}-\frac{F_{1} z(s)}{x(s)+a z(s)}\right] d s\right\}, \\
& y(t)=y_{0} \exp \left\{\int_{0}^{t}\left[\frac{\alpha F x(s)}{1+h F x(s)+w F y(s)}-\frac{b(s) z(s)}{D+z(s)}-d_{1}\right] d s\right\}, \\
& z(t)=z_{0} \exp \left\{\int_{0}^{t}\left[\frac{\beta b(s) y(s)}{D+z(s)}+\frac{F_{2} x(s)}{x(s)+a z(s)}-d_{2}-q(s) E\right] d s\right\} .
\end{aligned}
$$

The conclusion follows immediately for all $t \in[0, \infty)$.

Definition 4.1 System (3.5) is said to be permanent if there exist some $\delta_{i}>0$ $(i=1,2)$ with $0<\delta_{1}<\delta_{2}$ such that

$$
\begin{aligned}
& \min \left\{\lim _{t \rightarrow+\infty} \inf x(t), \lim _{t \rightarrow+\infty} \inf y(t), \lim _{t \rightarrow+\infty} \inf z(t)\right\} \geq \delta_{1}, \\
& \max \left\{\lim _{t \rightarrow+\infty} \sup x(t), \lim _{t \rightarrow+\infty} \sup y(t), \lim _{t \rightarrow+\infty} \sup z(t)\right\} \leq \delta_{2}
\end{aligned}
$$

for all solutions of system (3.5) with positive initial values. System (3.5) is said to be nonpersistent if there is a positive solution $(x(t), y(t), z(t))$ of (3.5) satisfying the following condition:

$$
\left.\min \left\{\lim _{t \rightarrow+\infty} \inf x(t), \lim _{t \rightarrow+\infty} \inf y(t), \lim _{t \rightarrow+\infty} \inf z(t)\right)\right\}=0 .
$$

Let $\epsilon \geq 0$ be sufficiently small. Put

$M_{1}^{\epsilon}=K+\epsilon$,

$M_{2}^{\epsilon}=\frac{\alpha F M_{1}^{\epsilon}-d_{1}\left(1+h F M_{1}^{\epsilon}\right)}{d_{1} w F}=\frac{M_{1}^{\epsilon}\left(\alpha-d_{1} h\right)}{d_{1} w}-\frac{1}{w F}, \quad m_{2}^{\epsilon}=\frac{\alpha F m_{1}^{\epsilon}-\left(b^{u}+d_{1}\right)\left(1+h F m_{1}^{\epsilon}\right)}{w F\left(b^{u}+d_{1}\right)}$,

$M_{3}^{\epsilon}=\frac{\beta b^{u} M_{2}^{\epsilon}+F_{2} D-D\left(d_{2}+q^{l} E\right)}{d_{2}+q^{l} E-F_{2}}=\frac{M_{2}^{\epsilon} \beta b^{u}}{d_{2}+q^{l} E-F_{2}}-D, \quad m_{3}^{\epsilon}=\frac{\beta b^{l} m_{2}^{\epsilon}}{d_{2}+q^{u} E}-D$,

then $M_{i}^{\epsilon}>m_{i}^{\epsilon}(i=1,2,3)$. We show that $\max \left\{m_{1}^{\epsilon}, 0\right\}, \max \left\{m_{2}^{\epsilon}, 0\right\}$ and $\max \left\{m_{3}^{\epsilon}, 0\right\}$ are the lower bounds for the limiting bounds of species $x(t), y(t)$ and $z(t)$, respectively as $t \rightarrow \infty$, which is obvious when $m_{i}^{\epsilon} \leq 0$. Thus, we assume that $m_{i}^{\epsilon}>0$, $(i=1,2,3)$.

\section{Lemma 4.2 Suppose}

$$
m_{i}^{0}>0, i=1,2,3
$$


then for any sufficiently small $\epsilon \geq 0$ such that $m_{i}^{\epsilon}>0(i=1,2,3)$, the region $\Gamma_{\epsilon}$ defined by

$$
\Gamma_{\epsilon}=\left\{(x, y, z) \in \mathbb{R}^{3} \mid m_{1}^{\epsilon} \leq x(t) \leq M_{1}^{\epsilon}, m_{2}^{\epsilon} \leq y(t) \leq M_{2}^{\epsilon}, m_{3}^{\epsilon} \leq z(t) \leq M_{3}^{\epsilon}\right\}(4.2)
$$
is positively invariant with respect to the system (3.5).

Geometrically, uniform permanence means the existence of a region in the phase plane at a non-zero distance from the boundary in which species enter and must lie ultimately that ensures the long time survival of species in biological sense.

Theorem 4.1 The system (3.5) is permanent provided the conditions in (3.1), (3.2) and (4.1) are satisfied.

Proof. Throughout this proof, we use the fact that solution to the equation

$$
X^{\prime}(t)=A(t, X) X(t)[B-X(t)],(B \neq 0)
$$

is given by

$$
X(t)=\frac{B X^{0} \exp \left\{\int_{0}^{t} B A(s, X(s)) d s\right\}}{X^{0}\left[\exp \left\{\int_{0}^{t} B A(s, X(s)) d s\right\}-1\right]+B}, X^{0}=X(0) .
$$

Consider the solution of system (2.1) with an initial value $\left(x_{0}, y_{0}, z_{0}\right) \in \Gamma_{\epsilon}$. From Lemma 4.1 and from the first equation of system (3.5), we have

$$
\dot{x}(t) \leq r x(t)\left(1-\frac{x(t)}{K}\right)=\frac{r}{K} x(t)(K-x(t)) \leq \frac{r}{K} x(t)\left\{M_{1}^{0}-x(t)\right\} .
$$

Using comparison test, we have

$$
x(t) \leq \frac{M_{1}^{0} x_{0} \exp (r t)}{x_{0}[\exp (r t)-1]+M_{1}^{0}} \leq \frac{M_{1}^{\epsilon} x_{0} \exp (r t)}{x_{0}[\exp (r t)-1]+M_{1}^{\epsilon}} \leq M_{1}^{\epsilon}, t \geq 0 .
$$

From the second equation of system (3.5), we have

$$
\begin{aligned}
\dot{y}(t) & \leq-d_{1} y(t)+\frac{\alpha F x(t) y(t)}{1+h F x(t)+w F y(t)} \\
& \leq-d_{1} y(t)+\frac{\alpha F M_{1}^{\epsilon} y(t)}{1+w F y(t)+h F M_{1}^{\epsilon}} \\
& =y(t)\left[-d_{1}+\frac{\alpha F M_{1}^{\epsilon}}{1+h F M_{1}^{\epsilon}+w F y(t)}\right] \\
& \leq \frac{d_{1} w F y(t)}{1+h F M_{1}^{\epsilon}+w F y(t)}\left[M_{2}^{\epsilon}-y(t)\right] .
\end{aligned}
$$

Let $c_{2}(t)=\frac{d_{1} w F}{1+h F M_{1}^{\epsilon}+w F y(t)}$. Using comparison test, we have

$$
y(t) \leq \frac{M_{2}^{\epsilon} y_{0} \exp \left\{M_{2}^{\epsilon} \int_{0}^{t} c_{2}(s) d s\right\}}{y_{0}\left[\exp \left\{M_{2}^{\epsilon} \int_{0}^{t} c_{2}(s) d s\right\}-1\right]+M_{2}^{\epsilon}} \leq M_{2}^{\epsilon}, t \geq 0 .
$$


From the third equation of system (3.5), we have

$$
\begin{aligned}
\dot{z}(t) & \leq \frac{\beta b^{u} M_{2}^{\epsilon}}{D+z(t)} z(t)+F_{2} z(t)-d_{2} z(t)-q^{l} E z(t) \\
& =z(t)\left\{\frac{\beta b^{u} M_{2}^{\epsilon}}{D+z(t)}+F_{2}-\left(d_{2}+q^{l} E\right)\right\} \\
& =\frac{z(t)}{D+z(t)}\left[\left\{\beta b^{u} M_{2}^{\epsilon}+F_{2} D-D\left(d_{2}+q^{l} E\right)\right\}-\left\{\left(d_{2}+q^{l} E\right)-F_{2}\right\} z(t)\right] \\
& \leq \frac{z(t)\left\{\left(d_{2}+q^{l} E\right)-F_{2}\right\}}{D+z(t)}\left[M_{3}^{\epsilon}-z(t)\right] .
\end{aligned}
$$

Let $c_{3}(t)=\frac{\left(d_{2}+q^{l} E\right)-F_{2}}{D+z(t)}$. Using comparison test, we have

$$
z(t) \leq \frac{M_{3}^{\epsilon} z_{0} \exp \left\{M_{3}^{\epsilon} \int_{0}^{t} c_{3}(s) d s\right\}}{z_{0}\left[\exp \left\{M_{3}^{\epsilon} \int_{0}^{t} c_{3}(s) d s\right\}-1\right]+M_{3}^{\epsilon}} \leq M_{3}^{\epsilon}, \forall t \geq 0 .
$$

Again, from the first equation of system (3.5), we have

$$
\begin{aligned}
\dot{x}(t) & \geq r x(t)\left(1-\frac{x(t)}{K}\right)-\frac{1}{w} x(t)-\frac{F_{1}}{a} x(t) \\
& =r x(t)\left[\left(1-\frac{1}{w}-\frac{F_{1}}{a}\right)-\frac{x(t)}{K}\right] \\
& \geq \frac{r}{K} x(t)\left[m_{1}^{0}-x(t)\right] .
\end{aligned}
$$

Since $x_{0}>m_{1}^{0}$, by comparison test, we have

$$
x(t) \geq \frac{m_{1}^{0} \exp \left\{r\left(1-\frac{a+w F_{1}}{a w}\right) t\right\}}{x_{0}\left[\exp \left\{r\left(1-\frac{a+w F_{1}}{a w}\right)\right\}-1\right]} \geq m_{1}^{\epsilon}, \forall t \geq 0 .
$$

From the second equation of system (3.5), we have

$$
\dot{y}(t) \geq y(t)\left[\frac{\alpha F m_{1}^{\epsilon}}{1+h F m_{1}^{\epsilon}+w F y(t)}-b^{u}-d_{1}\right] \geq \frac{\left(b^{u}+d_{1}\right) w F y(t)}{1+h F m_{1}^{\epsilon}+w F y(t)}\left[m_{2}^{\epsilon}-y(t)\right] .
$$

Let $c_{u}(t)=\frac{\left(b^{u}+d_{1}\right) w F}{1+h F m_{1}^{\epsilon}+w F y(t)}$. Using comparison test, we have

$$
y(t) \geq \frac{m_{2}^{\epsilon} y_{0} \exp \left\{m_{2}^{\epsilon} \int_{0}^{t} c_{4}(s) d s\right\}}{y_{0}\left[\exp \left\{m_{2}^{\epsilon} \int_{0}^{t} c_{4}(s) d s\right\}-1\right]+m_{2}^{\epsilon}} \geq m_{2}^{\epsilon}, \forall t \geq 0 .
$$

From the last equation of system (3.5), we have

$$
\dot{z}(t) \geq z(t)\left[\frac{\beta b^{l} m_{2}^{\epsilon}}{D+z(t)}-d_{2}-q^{u} E\right] \geq \frac{z(t)\left(d_{2}+q^{u} E\right)}{D+z(t)}\left[m_{3}^{\epsilon}-z(t)\right] .
$$

Since $z_{0} \geq m_{3}^{\epsilon}$, therefore $z(t) \geq m_{3}^{\epsilon}, \forall t \geq 0$. Hence, the region $\Gamma_{\epsilon}$ is positively invariant and consequently the system (3.5) is permanent. 
Remark 4.1 We established existence which leads to a criterion of species survival. It is based on the values of $b$ which is linked with the level of water in the lake. The result given by Theorem 4.1 has an interesting ecological interpretation, since it illustrates that suitable water levels can be an advantage in terms of species survival.

Theorem 4.2 If $M_{2}^{0}>0$ and $M_{3}^{0}<0$, then $\lim _{t \rightarrow \infty} z(t)=0$ i.e., the predator population goes to extinction.

Proof. Since $\dot{z}(t) \leq \frac{z(t)\left\{\left(d_{2}+q^{l} E\right)-F_{2}\right\}}{D+z(t)}\left[M_{3}^{\epsilon}-z(t)\right]<0$ (since $M_{3}^{0}<0 \Rightarrow$ $M_{3}^{\epsilon}<0$ with sufficiently small $\epsilon$ ). Thus, there exists $c \geq 0$ such that $\lim _{t \rightarrow \infty} z(t)=c$ and $c \leq z(t)<z_{0}, \forall t \geq 0$. If $c>0$, then there exists $\mu>0$ such that $z^{\prime}(t)<-\mu$, $\forall t \geq 0$. Therefore, $z(t)<-\mu t+z_{0}$ and $\lim _{t \rightarrow \infty} z(t)=-\infty$, which contradicts the fact that $z(t)>0, \forall t \geq 0$. Hence, $\lim _{t \rightarrow \infty} z(t)=0$.

Remark 4.2 The increased level of water induces zones inundation on the banks of the lake, which permits to have more invertebrates. Since invertebrates already exists in bush at the bank of reservoir, the invertebrates float on the surface of water due to increased water level. This promotes to have more prey, mathematically can be justified by $M_{2}^{0}>0$. These zones become an obstacle for the predator species because they are not deep and are protected from big predators; this hinders the capture of the prey by the predator. These areas are the privileged places for development of the prey. Consequently, if the level of water is high, which is directly associated with $M_{3}^{0}<0$, the predation could decrease significantly.

Theorem 4.3 If $M_{2}^{0} \leq 0$ and $d_{2}+q^{l} E>F_{2}$, then $\lim _{t \rightarrow \infty} y(t)=0$ and $\lim _{t \rightarrow \infty} z(t)=0$, that is, the prey and the predator goes to extinction.

Proof. It follows that $M_{2}^{0} \leq 0$ and $M_{3}^{0} \leq 0$. Thus, $\lim _{t \rightarrow \infty} z(t)=0$. Proof follows from the previous theorem.

Theorem 4.4 If the following conditions hold,

$$
M_{2}^{0} \leq 0, d_{2}+q^{l} E>F_{2}, 0<\frac{x_{0}}{y_{0}}<\frac{1}{h}\left[\frac{1}{r+b^{u}+d_{1}}-\left(w+\frac{1}{F M_{2}^{0}}\right)\right]=\delta,
$$

then $\lim _{t \rightarrow \infty}(x(t), y(t), z(t))=(0,0,0)$.

Proof. From the previous theorem, we have $\lim _{t \rightarrow \infty} y(t)=0$ and $\lim _{t \rightarrow \infty} z(t)=0$. First we assume that the condition holds and claim that $\frac{x(t)}{y(t)}<\delta, \forall t \geq 0$. If not, then there exists a first time $t_{1}$ such that $\frac{x\left(t_{1}\right)}{y\left(t_{1}\right)}=\delta$ and $\frac{x(t)}{y(t)}<\delta, \forall t \in\left[0, t_{1}\right]$. Then, for 
any $t \in\left[0, t_{1}\right]$, we have

$$
\begin{aligned}
\dot{x}(t) & \leq x(t)\left[r-\frac{F y(t)}{1+h F x(t)+w F y(t)}\right] \\
& =x(t)\left[r-\frac{F}{1 / y(t)+h F x(t) / y(t)+w F}\right] \\
& \leq x(t)\left[-b^{u}-d_{1}\right] .
\end{aligned}
$$

Thus, $x(t) \leq x_{0} \exp \left\{-t\left(b^{u}+d_{1}\right)\right\}$.

Again, from system (3.5), we have $\dot{y}(t) \geq y(t)\left[-b^{u}-d_{1}\right]$. Thus, $y(t) \geq$ $y_{0} \exp \left\{-\left(b^{u}+d_{1}\right) t\right\}$. Hence, $\frac{x(t)}{y(t)} \leq \frac{x_{0}}{y_{0}}<\delta, \forall t \in\left[0, t_{1}\right]$, which is contradiction to the existence of $t_{1}$. So, our claim is true. Since $x(t) \leq x_{0} \exp \left\{-\left(b^{u}+d_{1}\right) t\right\} \rightarrow 0$ as $t \rightarrow \infty$. Hence, $\lim _{t \rightarrow \infty}(x(t), y(t), z(t))=(0,0,0)$.

\section{Existence of positive periodic solutions}

In this section, we investigate the existence of positive periodic solutions of the system (3.5). Such a solution describes an equilibrium situation consistent with the variability of environmental conditions and such that populations survive. The trajectories in the phase plane of these solutions of the non-autonomous system take the place of the equilibrium points of the autonomous system.

To prove the existence of positive periodic solutions of system (3.5) with strictly positive components, we will employ the continuation theorem in coincidence degree theory, which has been successfully used to establish criteria for the existence of positive periodic solutions of some mathematical models $41,42,43$. To this end, we shall summarize in the following a few concepts and results from ${ }^{37}$ that will be basic for this section.

Let $X$ and $Z$ be two real Banach spaces and $L: \operatorname{Dom} L \subset X \rightarrow Z$ a linear mapping, and $N: X \rightarrow Z$ a continuous mapping. The mapping $L$ is called a Fredholm mapping of index zero if $\operatorname{dimKer} L=\operatorname{codim} \operatorname{Im} L<+\infty$, and $\operatorname{Im} L$ is closed in $Z$. If $L$ is a Fredholm mapping of index zero, there exist continuous projections $P: X \rightarrow X$ and $Q: Z \rightarrow Z$ such that $\operatorname{Im} P=\operatorname{Ker} L, \operatorname{Im} L=\operatorname{Ker} Q=\operatorname{Im}(I-Q)$. It follows that $\left.L\right|_{\operatorname{Dom} L \cap \operatorname{Ker} P}:(I-P) X \rightarrow \operatorname{Im} L$ has an inverse which will be denoted by $K_{P}$. If $\Omega$ is an open and bounded subset of $X$, the mapping $N$ will be called $L$-compact on $\bar{\Omega}$ if $Q N(\bar{\Omega})$ is bounded and $K_{P}(I-Q) N: \bar{\Omega} \rightarrow X$ is compact. Since $\operatorname{Im} Q$ is isomorphic to $\operatorname{Ker} L$, there exists an isomorphism $J: \operatorname{Im} Q \rightarrow \operatorname{Ker} L$.

In the proof of our main theorem, we will use the following result from Gaines and Mawhin ${ }^{37}$.

Theorem 5.1 [Continuation Theorem] Let $\Omega \subset X$ be an open bounded set. Let $L$ be a Fredholm mapping of index zero and $N$ be L-compact on $\bar{\Omega}$. Suppose that

(1) For each $\lambda \in(0,1), u \in \partial \Omega \cap D o m L, L u \neq \lambda N u$;

(2) For each $u \in \partial \Omega \cap \operatorname{Ker} L, Q N u \neq 0$; 
(3) The Brouwer degree, $\operatorname{deg}\{J Q N, \Omega \cap K \operatorname{KerL}, 0\} \neq 0$.

Then the equation $L u=N u$ has at least one solution in $\bar{\Omega} \cap$ DomL.

Theorem 5.2 System (3.5) has atleast one positive periodic solutions if the following conditions are satisfied,

$$
\begin{aligned}
& K\left[1-\frac{1}{r}\left(\frac{1}{w}+\frac{F_{1}}{a}\right)\right]>0, \\
& \alpha F e^{\rho_{2}}-\left(1+h F e^{\rho_{2}}\right)\left(d_{1}+b_{2}\right)>0, \\
& \frac{\beta b_{1} e^{\rho_{4}}}{d_{2}+q^{M} E}-D>0 .
\end{aligned}
$$

Proof.

System (3.5) has atleast one positive periodic solution as solution of the system remains positive for $t \geq 0$. Let

$$
U(t)=\log x(t), V(t)=\log y(t), W(t)=\log z(t),
$$

then we have

$$
\begin{aligned}
\dot{U}(t) & =r-\frac{r e^{U(t)}}{K}-\frac{F e^{V(t)}}{1+h F e^{U(t)}+w F e^{V(t)}}-\frac{F_{1} e^{W(t)}}{e^{U(t)}+a e^{W(t)}}, \\
\dot{V}(t) & =\frac{\alpha F e^{U(t)}}{1+h F e^{U(t)}+w F e^{V(t)}}-\frac{b(t) e^{W(t)}}{D+e^{W(t)}}-d_{1}, \\
\dot{W}(t) & =\frac{\beta b(t) e^{V(t)}}{D+e^{W(t)}}+\frac{F_{2} e^{U(t)}}{e^{U(t)}+a e^{W(t)}}-d_{2}-q(t) E .
\end{aligned}
$$

In order to use Continuation Theorem on system (5.2), we take

$$
X=Z=\left\{S \in(U, V, W)^{T} \in \mathbb{C}\left(\mathbb{R}, \mathbb{R}^{3}\right), S(t+1)=S(t)\right\}
$$

and use the norm

$$
\|S\|=\max _{t \in[0,1]}|U(t)|+\max _{t \in[0,1]}|V(t)|+\max _{t \in[0,1]}|W(t)|, S \in X(\text { or } Z) .
$$

Then, $X$ and $Z$ are the Banach spaces when they are endowed with the above norm ||. |l.

Let

$$
N\left[\begin{array}{c}
U \\
V \\
W
\end{array}\right]=\left[\begin{array}{l}
N_{1}(t) \\
N_{2}(t) \\
N_{3}(t)
\end{array}\right]=\left[\begin{array}{c}
r-\frac{r e^{U(t)}}{K}-\frac{F e^{V(t)}}{1+h F e^{U(t)}+w F e^{V(t)}}-\frac{F_{1} e^{W(t)}}{e^{U(t)}+a e^{W(t)}} \\
\frac{\alpha F e^{U(t)}}{1+h F e^{U(t)}+w F e^{V(t)}}-\frac{b(t) e^{W(t)}}{D+e^{W(t)}}-d_{1} \\
\frac{\beta b(t) e^{V(t)}}{D+e^{W(t)}}+\frac{F_{2} e^{U(t)}}{e^{U(t)}+a e^{W(t)}}-q(t) E-d_{2}
\end{array}\right]
$$


and

$$
L\left(\begin{array}{c}
U \\
V \\
W
\end{array}\right)=\left(\begin{array}{c}
\dot{U} \\
\dot{V} \\
\dot{W}
\end{array}\right), P\left(\begin{array}{c}
U \\
V \\
W
\end{array}\right)=Q\left(\begin{array}{c}
U \\
V \\
W
\end{array}\right)=\left(\begin{array}{c}
\int_{0}^{1} U(t) d t \\
\int_{0}^{1} V(t) d t \\
\int_{0}^{1} W(t) d t
\end{array}\right) .
$$

Here, $P$ and $Q$ are continuous projections, and

$$
\left(\begin{array}{c}
U \\
V \\
W
\end{array}\right) \in X \text { such that } \operatorname{Im} P=\operatorname{Ker} L, \operatorname{Ker} Q=\operatorname{Im} L=\operatorname{Im}(I-Q) .
$$

Then,

$$
\begin{aligned}
\operatorname{Ker} L & =\left\{(U, V, W) \in X:(U, V, W)=\left(h_{1}, h_{2}, h_{3}\right) \in \mathbb{R}^{3}\right\}=\mathbb{R}^{3}, \\
\operatorname{Im} L & =\left\{(U, V, W) \in Z: \int_{0}^{1} U(t) d t=0, \quad \int_{0}^{1} V(t) d t=0, \quad \int_{0}^{1} W(t) d t=0\right\} .
\end{aligned}
$$

Here, $\operatorname{dimKer} L=3=\operatorname{Codim} \operatorname{Im} L$. Since $\operatorname{Im} L$ is closed, hence $L$ is a Fredholm mapping zero. So, we define inverse of $L$ as

$$
K_{p}: \operatorname{Im} L \rightarrow \operatorname{Dom} L \cap \operatorname{Ker} P
$$

given by

$$
K_{p}\left(\begin{array}{c}
U \\
V \\
W
\end{array}\right)=\left[\begin{array}{c}
\int_{0}^{t} U(s) d s-\int_{0}^{1} \int_{0}^{t} U(s) d s d t \\
\int_{0}^{t} V(s) d s-\int_{0}^{1} \int_{0}^{t} V(s) d s d t \\
\int_{0}^{t} W(s) d s-\int_{0}^{1} \int_{0}^{t} W(s) d s d t
\end{array}\right] .
$$

Accordingly, $Q N: X \rightarrow Z$ and $K_{p}(I-Q) N: X \rightarrow X$ lead to

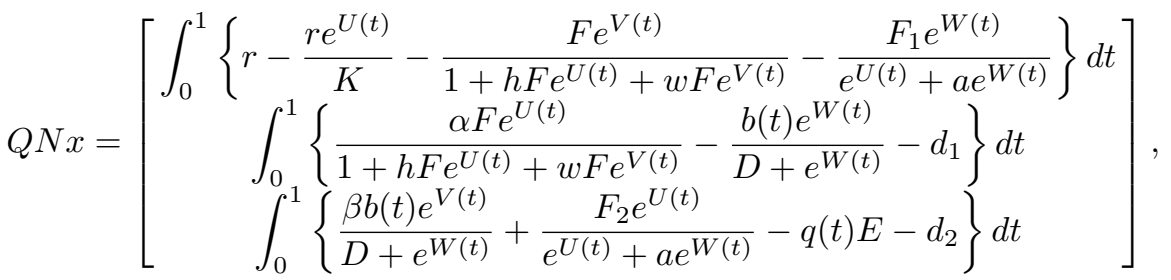

$$
\begin{aligned}
& K_{p}(I-Q) N\left(\begin{array}{c}
U \\
V \\
W
\end{array}\right)=\left[\begin{array}{l}
\int_{0}^{t} N_{1}(s) d s-\int_{0}^{1} \int_{0}^{t} N_{1}(s) d s d t-\left(t-\frac{1}{2}\right) \int_{0}^{1} N_{1}(s) d s \\
\int_{0}^{t} N_{2}(s) d s-\int_{0}^{1} \int_{0}^{t} N_{2}(s) d s d t-\left(t-\frac{1}{2}\right) \int_{0}^{1} N_{2}(s) d s \\
\int_{0}^{t} N_{3}(s) d s-\int_{0}^{1} \int_{0}^{t} N_{3}(s) d s d t-\left(t-\frac{1}{2}\right) \int_{0}^{1} N_{3}(s) d s
\end{array}\right] .
\end{aligned}
$$


Using Arzela-Ascoli theorem $Q N(\bar{\Omega})$ and $K_{p}(I-Q) N(\bar{\Omega})$ are relatively compact for any bounded open set $\Omega \subset X$. Thus, $N$ is $L$-compact on any open bounded set $X$.

In order to apply Continuation Theorem, we need a suitable open bounded subset $\Omega$ corresponding to the operator equation $L x=\lambda N x, \lambda \in(0,1)$. We have

$$
\begin{aligned}
\dot{U}(t) & =\lambda\left[r-\frac{r e^{U(t)}}{K}-\frac{F e^{V(t)}}{1+h F e^{U(t)}+w F e^{V(t)}}-\frac{F_{1} e^{W(t)}}{e^{U(t)}+a e^{W(t)}}\right], \\
\dot{V}(t) & =\lambda\left[\frac{\alpha F e^{U(t)}}{1+h F e^{U(t)}+w F e^{V(t)}}-\frac{b(t) e^{W(t)}}{D+e^{W(t)}}-d_{1}\right], \\
\dot{W}(t) & =\lambda\left[\frac{\beta b(t) e^{V(t)}}{D+e^{W(t)}}+\frac{F_{2} e^{U(t)}}{e^{U(t)}+a e^{W(t)}}-q(t) E-d_{2}\right] .
\end{aligned}
$$

Assume that $(U, V, W)^{T} \in X$ is an arbitrary solution of system (5.3) for certain $\lambda \in(0,1)$. Integrating both sides of equations in system $(5.3)$ over $[0,1]$, we get

$$
\begin{aligned}
r & =\frac{r}{K} \int_{0}^{1} e^{U(t)} d t+F \int_{0}^{1} \frac{e^{V(t)}}{1+h F e^{U(t)}+w F e^{V(t)}} d t+F_{1} \int_{0}^{1} \frac{e^{W(t)}}{e^{U(t)}+a e^{W(t)}} d t, \\
d_{1} & =\alpha F \int_{0}^{1} \frac{e^{U(t)}}{1+h F e^{U(t)}+w F e^{V(t)}} d t-\int_{0}^{1} \frac{b(t) e^{W(t)}}{D+e^{W(t)}} d t \\
d_{2} & =\beta \int_{0}^{1} \frac{b(t) e^{V(t)}}{D+e^{W(t)}} d t+F_{2} \int_{0}^{1} \frac{e^{U(t)} d t}{e^{U(t)}+a e^{W(t)}} d t-E \int_{0}^{1} q(t) d t .
\end{aligned}
$$

From (5.3) and (5.4), we have

$$
\begin{gathered}
\int_{0}^{1}|\dot{U}(t)|=\lambda \int_{0}^{1} \mid r-\frac{r e^{U(t)}}{K}-\frac{F e^{V(t)}}{1+h F e^{U(t)}+w F e^{V(t)}} \\
\quad-\frac{F_{1} e^{W(t)}}{e^{U(t)}+a e^{W(t)}} \mid d t \leq 2 r \\
\int_{0}^{1}|\dot{V}(t)|=\lambda \int_{0}^{1}\left|\frac{\alpha F e^{U(t)}}{1+h F e^{U(t)}+w F e^{V(t)}}-\frac{b(t) e^{W(t)}}{D+e^{W(t)}}-d_{1}\right| d t \leq 2 d_{1}, \\
\int_{0}^{1}|\dot{W}(t)|=\lambda \int_{0}^{1}\left|\frac{\beta b(t) e^{V(t)}}{D+e^{W(t)}}+\frac{F_{2} e^{U(t)}}{e^{U(t)}+a e^{W(t)}}-q(t) E-d_{2}\right| d t \leq 2 d_{2} .
\end{gathered}
$$

So, there exist $\xi_{i}, \eta_{i} \in[0,1]$ such that for $t \in[0,1]$, we have

$$
\begin{aligned}
& U\left(\xi_{1}\right)=\min U(t), U\left(\eta_{1}\right)=\max U(t), V\left(\xi_{2}\right)=\min V(t), V\left(\eta_{2}\right)=\max V(t), \\
& W\left(\xi_{3}\right)=\min W(t), W\left(\eta_{3}\right)=\max W(t) .
\end{aligned}
$$

From (5.4) and (5.8), we have

$r>\frac{r}{K} \int_{0}^{1} U(t) d t>\frac{r}{K} \exp \left\{U\left(\xi_{1}\right)\right\} \Rightarrow K>\exp \left\{U\left(\xi_{1}\right)\right\} \Rightarrow U\left(\xi_{1}\right) \leq \log (K), \forall t \geq 0$.

Hence, $U(t)<U\left(\xi_{1}\right)+\int_{0}^{1}|\dot{U}(t)| \leq \log (K)+2 r=\rho_{1} \geq 0$. 
Again, from (5.3) and (5.8), we have

$$
r \leq \frac{r}{K} \int_{0}^{1} e^{U(t)} d t+F \int_{0}^{1} \frac{e^{V(t)}}{w F e^{V(t)}} d t+F_{1} \int_{0}^{1} \frac{e^{W(t)}}{a e^{W(t)}} \leq \frac{r}{K} e^{\eta_{1}}+\left(\frac{1}{w}+\frac{F_{1}}{a}\right) .
$$

This implies,

$$
e^{U\left(\eta_{1}\right)} \geq \frac{K}{r}\left[r-\left(\frac{1}{w}+\frac{F_{1}}{a}\right)\right] \Rightarrow U\left(\eta_{1}\right) \geq \log \left[K\left\{1-\frac{1}{r}\left(\frac{1}{w}+\frac{F_{1}}{a}\right)\right\}\right] .
$$

Thus, $U(t) \geq U\left(\eta_{1}\right)-\int_{0}^{1}|\dot{U}(t)| d t \geq \log \left[K\left\{1-\frac{1}{r}\left(\frac{1}{w}+\frac{F_{1}}{a}\right)\right\}\right]-2 r=\rho_{2} \geq 0$.

Now take $\max |U(t)| \leq \max \left\{\rho_{1}, \rho_{2}\right\}=B_{1}$.

Again, from (5.4) and (5.8), we have

$$
d_{1} \leq \alpha F \int_{0}^{1} \frac{e^{U(t)}}{1+h F e^{U(t)}+w F e^{V(t)}} d t \leq \alpha F \frac{e^{\rho_{1}}}{1+h F e^{\rho_{1}}+w F e^{V\left(\xi_{2}\right)}} .
$$

This implies,

$$
e^{V\left(\xi_{2}\right)} \leq\left(\frac{\alpha F e^{\rho_{1}}}{d_{1}}-h F e^{\rho_{1}}-1\right) \Rightarrow V\left(\xi_{2}\right) \leq \log \left[\frac{\alpha F e^{\rho_{1}}-d_{1}\left(h F e^{\rho_{1}}+1\right)}{d_{1}}\right] .
$$

It follows that

$$
V(t) \leq V\left(\xi_{2}\right)+\int_{0}^{1}|\dot{V}(t)| d t \leq \log \left[\frac{\alpha F e^{\rho_{1}}-d_{1}\left(h F e^{\rho_{1}}+1\right)}{d_{1}}\right]+2 d_{1}=\rho_{3}, \forall t \geq 0 .
$$

Again from (5.4) and (5.8), we have

$$
d_{1} \geq \frac{\alpha F e^{\rho_{2}}}{1+h F e^{\rho_{2}}+w F e^{V\left(\eta_{2}\right)}}-b^{u} .
$$

Thus,

$\left(d_{1}+b^{u}\right)\left(1+h F e^{\rho_{2}}+w F e^{V\left(\eta_{2}\right)}\right) \geq \alpha F e^{\rho_{2}} \Rightarrow V\left(\eta_{2}\right) \geq \log \left[\frac{\alpha F e^{\rho_{2}}-\left(1+h F e^{\rho_{2}}\right)\left(d_{1}+b^{u}\right)}{\left(d_{1}+b^{u}\right) w F}\right]$.

So,

$V(t) \geq V\left(\eta_{2}\right)-\int_{0}^{1}|\dot{V}(t)| d t \geq \log \left(\frac{\alpha F e^{\rho_{2}}-\left(1+h F e^{\rho_{2}}\right)\left(d_{1}+b^{u}\right)}{\left(d_{1}+b^{u}\right) w F}\right)-2 d_{1}=\rho_{4}, \quad \forall t \geq 0$.

Now take $\max _{t \in[0,1]}|V(t)| \leq \max \left\{\left|\rho_{3}\right|,\left|\rho_{4}\right|\right\}=B_{2}$.

Again from (5.4) and (5.8), we have

$$
\begin{aligned}
& d_{2} \leq \frac{\beta b^{u} e^{\rho_{3}}}{D+e^{W\left(\xi_{3}\right)}}+\left(F_{2}-q^{l} E\right) \\
& \Rightarrow e^{W\left(\xi_{3}\right)} \leq\left(\frac{\beta b^{u} e^{\rho_{3}}}{d_{2}+q^{l} E-F_{2}}-D\right) \Rightarrow W\left(\xi_{3}\right) \leq \log \left(\frac{\beta b^{u} e^{\rho_{3}}}{d_{2}+q^{l} E-F_{2}}-D\right) .
\end{aligned}
$$

Hence,

$$
W(t) \leq W\left(\xi_{3}\right)+\int_{0}^{1}|\dot{W}(t)| d t \leq \log \left(\frac{\beta b_{2} e^{\rho_{3}}}{d_{2}+q^{m} E-F_{2}}-D\right)+2 d_{2}=\rho_{5} .
$$


Again, we have

$$
\begin{aligned}
& d_{2} \geq \frac{\beta b^{l} e^{\rho_{4}}}{D+e^{W\left(\eta_{3}\right)}}-q^{u} E \\
& \Rightarrow D+e^{W\left(\eta_{3}\right)} \geq \frac{\beta b^{l} e^{\rho_{4}}}{d_{2}+q^{u} E} \Rightarrow W\left(\eta_{3}\right) \geq \log \left[\frac{\beta b^{l} e^{\rho_{4}}}{d_{2}+q^{u} E}-D\right] .
\end{aligned}
$$

Hence,

$$
W(t) \geq W\left(\eta_{3}\right)-\int_{0}^{1}|\dot{W}(t)| d t=\log \left(\frac{\beta b^{l} e^{\rho_{4}}}{d_{2}+q^{u} E}-D\right)-2 d_{2}=\rho_{6} .
$$

Therefore, $\max _{t \in[0,1]}|W(t)| \leq \max \left\{\left|\rho_{5}\right|,\left|\rho_{6}\right|\right\}=B_{3}$.

Let $B=B_{1}+B_{2}+B_{3}+\epsilon$, where $\epsilon$ is chosen sufficiently large such that each solution $\left(U^{*}, V^{*}, W^{*}\right)^{T}$ of system of algebraic equations $\left[\bar{f}=\int_{0}^{1} f(t)\right]$,

$$
\begin{aligned}
& r-\frac{r e^{U}}{K}-\frac{F e^{V}}{1+h F e^{U}+w F e^{V}}-\frac{F_{1} e^{W}}{e^{U}+a e^{W}}=0, \\
& \frac{\alpha F}{1+h F e^{U}+w F e^{V}}-\frac{\bar{b} e^{W}}{D+e^{W}}-d_{1}=0, \\
& \frac{\beta \bar{b} e^{V}}{D+e^{W}}+\frac{F_{2} e^{U}}{e^{U}+a e^{W}}-\bar{q} E-d_{2}=0
\end{aligned}
$$

satisfies $\left\|\left(U^{*}, V^{*}, W^{*}\right)^{T}\right\|<B$ provided the system (5.9) has one or a number of solutions.

Now, we set $\Omega=\left\{(U, V, W)^{T} \in X \mid\left\|(U, V, W)^{T}\right\|<B\right\}$. It is easy to see that the first condition of Continuation Theorem is satisfied. Then, $(U, V, W)^{T} \in$ $\partial \Omega \cap \operatorname{Ker} L=\partial \Omega \cap \mathbb{R}^{3},(U, V, W)^{T}$ is content vector in $\mathbb{R}^{3}$ with $|U|+|V|+|W|=B$.

If the system (5.9) has atleast one solution, we have

$$
Q N\left(\begin{array}{c}
U \\
V \\
W
\end{array}\right)=\left(\begin{array}{c}
r-\frac{r e^{U}}{K}-\frac{F e^{V}}{1+h F e^{U}+w F e^{V}}-\frac{F_{1} e^{W}}{e^{U}+a e^{W}} \\
\frac{\alpha F}{1+h F e^{U}+w F e^{V}}-\frac{\bar{b} e^{W}}{D+e^{W}}-d_{1} \\
\frac{\beta \bar{b} e^{V}}{D+e^{W}}+\frac{F_{2} e^{U}}{e^{U}+a e^{W}}-\bar{q} E-d_{2}
\end{array}\right) \neq\left(\begin{array}{l}
0 \\
0 \\
0
\end{array}\right) .
$$

If the system (5.9) does not have any solution, we have

$$
Q N\left(\begin{array}{c}
U \\
V \\
W
\end{array}\right) \neq\left(\begin{array}{l}
0 \\
0 \\
0
\end{array}\right)
$$

Hence, the second condition of Continuation Theorem is satisfied.

In order to prove the third condition of Continuation Theorem, we define a homomorphism mapping $J: \operatorname{Im} Q \rightarrow \operatorname{Ker} L$ by $(U, V, W)^{T} \rightarrow(U, V, W)^{T}$. A standard 
and straight forward calculation shows that

$$
\begin{gathered}
\operatorname{deg}\left(J Q N(U, V, W)^{T}, \Omega \cap \operatorname{Ker} L,(0,0,0)^{T}\right)=\sum_{Z_{i}^{*} \in Q N^{-1}(0)} \operatorname{sgn} J Q N\left(Z_{i}^{*}\right) \\
=\sum_{\left(U_{i}^{*}, V_{i}^{*}, W_{i}^{*}\right) \in Q N^{-1}(0)} \operatorname{det} G\left(U^{*}, V^{*}, W^{*}\right) \prod_{i=1}^{n} U_{i}^{*} \prod_{i=1}^{n} V_{i}^{*} \prod_{i=1}^{n} W_{i}^{*} \neq 0,
\end{gathered}
$$

where $G(U, V, W)$ is a $3 \times 3$ matrix $G$ whose components are obtained from the system of algebraic equations (5.9) and given as,

$$
\begin{aligned}
& G_{11}=-\frac{r e^{U}}{K}+\frac{h F^{2} e^{U+V}}{\left(1+h F e^{U}+w F e^{V}\right)^{2}}+\frac{F_{1} e^{U+W}}{\left(e^{U}+a e^{W}\right)^{2}}, G_{12}=-\frac{F e^{V}\left(1+h F e^{U}\right)}{\left(1+h F e^{U}+w F e^{V}\right)^{2}}, \\
& G_{13}=-\frac{F_{1} e^{U+W}}{\left(e^{U}+a e^{W}\right)^{2}}, G_{21}=\frac{\alpha F e^{U}\left(1+w F e^{V}\right)}{\left(1+h F e^{U}+w F e^{V}\right)^{2}}, G_{22}=-\frac{\alpha w F^{2} e^{U+V}}{\left(1+h F e^{U}+w F e^{V}\right)^{2}}, \\
& G_{23}=\frac{D \bar{b} e^{W}}{\left(D+e^{W}\right)^{2}}, G_{31}=\frac{a F_{2} e^{U+W}}{\left(e^{U}+a e^{W}\right)^{2}}, G_{32}=\frac{\beta \bar{b} e^{V}\left(D+e^{W}\right)}{\left(D+e^{W}\right)^{2}}, \\
& G_{33}=-\frac{\beta \bar{b} e^{V+W}}{\left(D+e^{W}\right)^{2}}-\frac{a F_{2} e^{U+W}}{\left(e^{U}+a e^{W}\right)^{2}} .
\end{aligned}
$$

Hence, the third condition of Continuation Theorem is satisfied. Therefore, the system (5.2) has atleast one positive periodic solution. Hence, the system (3.5) has atleast one positive periodic solution.

Lemma 5.1 Let $\kappa$ be a real number and $f$ be a nonnegative function defined on $[\kappa,+\infty)$ such that $f$ is integrable on $[\kappa,+\infty)$ and is uniformly continuous on $[\kappa,+\infty)$, then $\lim _{t \rightarrow+\infty} f(t)=0$.

Definition 5.1 If $\widetilde{x}(t)$ is a $\omega$-periodic solution of the system (2.1), and $x(t)$ is any solution of the system (2.1) satisfying $\lim _{t \rightarrow \infty}|\widetilde{x}(t)-x(t)|=0$, then the $\omega$-periodic solution $\widetilde{x}(t)$ is said to be globally attractive.

\section{Global attractivity of positive periodic solution}

Theorem 6.1 If the system (3.5) has atleast one positive periodic solution and $0<x_{0}, y_{0}, z_{0}<\infty$, then the system (3.5) has unique positive periodic solution 
which is globally attractive provided the following conditions are satisfied:

$$
\begin{gathered}
\frac{\mu_{1} r}{K}+\frac{\mu_{1} h F^{2} e^{\rho_{4}}}{\left(1+h F e^{\rho_{1}}+w F e^{\rho_{3}}\right)^{2}}>\frac{\mu_{1} F_{1} e^{\rho_{5}}}{\left(e^{\rho_{2}}+a e^{\rho_{6}}\right)^{2}}+\frac{\mu_{2} \alpha F\left(1+F w e^{\rho_{3}}\right)}{\left(1+h F e^{\rho_{2}}+w F e^{\rho_{4}}\right)^{2}} \\
+\frac{\mu_{3} F_{2} e^{\rho_{5}}}{\left(e^{\rho_{2}}+a e^{\rho_{6}}\right)^{2}}, \\
\frac{F^{2} e^{\rho_{2}}\left(\mu_{2} \alpha w+\mu_{1} h\right)}{\left(1+h F e^{\rho_{1}}+w F e^{\rho_{3}}\right)^{2}}>\frac{\mu_{1} F}{\left(1+h F e^{\rho_{2}}+w F e^{\rho_{4}}\right)^{2}}+\frac{\mu_{3} \beta b^{u} e^{\rho_{5}}}{\left(D+e^{\rho_{6}}\right)^{2}} \\
\quad+\frac{\mu_{3} \beta b^{u}}{D+e^{\rho_{6}}}, \\
\frac{\mu_{3} F_{2} e^{\rho_{2}}}{\left(e^{\rho_{2}}+a e^{\rho_{6}}\right)^{2}}>\frac{\mu_{1} F_{1} e^{\rho_{1}}}{\left(e^{\rho_{1}}+a e^{\rho_{5}}\right)^{2}}+\frac{\mu_{2} b^{u} e^{\rho_{5}}}{\left(D+e^{\rho_{6}}\right)^{2}}+\frac{\mu_{2} b^{u}}{D+e^{\rho_{6}}}+\frac{\mu_{3} \beta b_{2} e^{\rho_{3}}}{\left(D+e^{\rho_{6}}\right)^{2}} .
\end{gathered}
$$

\section{Proof.}

The system (3.5) has atleast one positive periodic solution $(\widetilde{x}(t), \widetilde{y}(t), \widetilde{z}(t))$ and also we have

$$
e^{\rho_{2}} \leq \widetilde{x}(t) \leq e^{\rho_{1}}, e^{\rho_{4}} \leq \widetilde{y}(t) \leq e^{\rho_{3}}, e^{\rho_{6}} \leq \widetilde{z}(t) \leq e^{\rho_{5}}
$$

Suppose $(x(t), y(t), z(t))$ be any positive periodic solution. Let

$$
V(t)=\mu_{1}|\ln x(t)-\ln \widetilde{x}(t)|+\mu_{2}|\ln y(t)-\ln \widetilde{y}(t)|+\mu_{3}|\ln z(t)-\ln \widetilde{z}(t)|
$$

By calculating Dini's derivative, we get

$$
\begin{gathered}
D^{+} V(t)=\mu_{1} \operatorname{sgn}(x(t)-\widetilde{x}(t))\left(\frac{\dot{x}(t)}{x(t)}-\frac{\dot{\tilde{x}}(t)}{\widetilde{x}(t)}\right)+\mu_{2} \operatorname{sgn}(y(t)-\widetilde{y}(t))\left(\frac{\dot{y}(t)}{y(t)}-\frac{\dot{\tilde{y}}(t)}{\widetilde{y}(t)}\right) \\
+\mu_{3} \operatorname{sgn}(z(t)-\widetilde{z}(t))\left(\frac{\dot{z}(t)}{z(t)}-\frac{\dot{\widetilde{z}}(t)}{\widetilde{z}(t)}\right) .
\end{gathered}
$$


We have,

$$
\begin{aligned}
\mu_{1} \operatorname{sgn}( & x(t)-\widetilde{x}(t))\left(\frac{\dot{x}(t)}{x(t)}-\frac{\dot{\tilde{x}}(t)}{\widetilde{x}(t)}\right) \leq \mu_{1}\left[\left\{\frac{F_{1} z(t)}{(x(t)+a z(t))(\widetilde{x}(t)+a \widetilde{z}(t))}-\frac{r}{K}\right.\right. \\
& \left.-\frac{h F^{2} y(t)}{(1+h F x(t)+w F y(t))(1+h F \widetilde{x}(t)+w F \widetilde{y}(t))}\right\}|x(t)-\widetilde{x}(t)| \\
& +\frac{F(1-h F x(t))}{(1+h F x(t)+w F y(t))(1+h F \widetilde{x}(t)+w F \widetilde{y}(t))}|y(t)-\widetilde{y}(t)| \\
& \left.+\frac{F_{1} x(t)}{(x(t)+a z(t))(\widetilde{x}(t)+a \widetilde{z}(t))}|z(t)-\widetilde{z}(t)|\right], \\
\mu_{2} \operatorname{sgn}( & y(t)-\widetilde{y}(t))\left(\frac{\dot{y}(t)}{y(t)}-\frac{\dot{\widetilde{y}}(t)}{\widetilde{y}(t)}\right) \leq \mu_{2}\left[\frac{\alpha w F^{2} x(t)}{(1+h F x(t)+w F y(t))(1+h F \widetilde{x}(t)+w F \widetilde{y}(t))}|x(t)-\widetilde{x}(t)|\right. \\
& -\frac{{ }^{2}}{(1+h F x(t)+w F y(t))(1+h F \widetilde{x}(t)+w F \widetilde{y}(t))}|y(t)-\widetilde{y}(t)| \\
& \left.+\frac{b(t)(D+2 z(t))}{(D+z(t))(D+\widetilde{z}(t))}|z(t)-\widetilde{z}(t)|\right], \\
\mu_{3} \operatorname{sgn}( & z(t)-\widetilde{z}(t))\left(\frac{\dot{z}(t)}{z(t)}-\frac{\dot{\tilde{z}}(t)}{\widetilde{z}(t)}\right) \leq \mu_{3}\left[\frac{F_{2} z(t)}{(x(t)+a z(t))(\widetilde{x}(t)+a \widetilde{z}(t))}|x(t)-\widetilde{x}(t)|\right. \\
& +\frac{\beta b(t)(D+2 z(t))}{(D+z(t))(D+\widetilde{z}(t))}|y(t)-\widetilde{y}(t)| \\
& \left.+\left\{\frac{\beta b(t) y(t)}{(D+z(t))(D+\widetilde{z}(t))}-\frac{F_{2} x(t)}{(x(t)+a z(t))(\widetilde{x}(t)+a \widetilde{z}(t))}\right\}|z(t)-\widetilde{z}(t)|\right] .
\end{aligned}
$$

Thus, we have

$$
\begin{aligned}
D^{+} V(t) \leq-\left[\mu_{1}\right. & \left\{\frac{r}{K}+\frac{h F^{2} y(t)}{(1+h F x(t)+w F y(t))(1+h F \widetilde{x}(t)+w F \widetilde{y}(t))}-\frac{F_{1} z(t)}{(x(t)+a z(t))(\widetilde{x}(t)+a \widetilde{z}(t))}\right\} \\
& -\mu_{2} \frac{\alpha F(1+w F y(t))}{(1+h F x(t)+w F y(t))(1+h F \widetilde{x}(t)+w F \widetilde{y}(t))} \\
& \left.-\mu_{3} \frac{F_{2} z(t)}{(x(t)+a z(t))(\widetilde{x}(t)+a \widetilde{z}(t))}\right]|x(t)-\widetilde{x}(t)| \\
- & \quad \mu_{1} \frac{F(h F x(t)-1)}{(1+h F x(t)+w F y(t))(1+h F \widetilde{x}(t)+w F \widetilde{y}(t))} \\
& +\mu_{2} \frac{\alpha w F^{2} x(t)}{(1+h F x(t)+w F y(t))(1+h F \widetilde{x}(t)+w F \widetilde{y}(t))} \\
& \left.-\mu_{3} \frac{\beta b(t)(D+2 z(t))}{(D+z(t))(D+\widetilde{z}(t))}\right]|y(t)-\widetilde{y}(t)| \\
- & -\mu_{1} \frac{F_{1} x(t)}{(x(t)+a z(t))(\widetilde{x}(t)+a \widetilde{z}(t))}-\mu_{2} \frac{b(t)(D+2 z(t))}{(D+z(t))(D+\widetilde{z}(t))} \\
& \left.+\mu_{3}\left\{\frac{F_{2} x(t)}{(x(t)+a z(t))(\widetilde{x}(t)+a \widetilde{z}(t))}-\frac{\beta b(t) y(t)}{(D+z(t))(D+\widetilde{z}(t))}\right\}\right]|z(t)-\widetilde{z}(t)| .
\end{aligned}
$$


Therefore,

$$
D^{+} V(t) \leq-\delta_{1}|x(t)-\widetilde{x}(t)|-\delta_{2}|y(t)-\widetilde{y}(t)|-\delta_{3}|z(t)-\widetilde{z}(t)|,
$$

where

$$
\begin{aligned}
& \delta_{1}=\frac{\mu_{1} r}{K}+\frac{\mu_{1} h F^{2} e^{\rho_{4}}}{\left(1+h F e^{\rho_{1}}+w F e^{\rho_{3}}\right)^{2}}-\frac{\mu_{1} F_{1} e^{\rho_{5}}}{\left(e^{\rho_{2}}+a e^{\rho_{6}}\right)^{2}}-\frac{\mu_{2} \alpha F\left(1+w F e^{\rho_{3}}\right)}{\left(1+h F e^{\rho_{2}}+w F e^{\rho_{4}}\right)^{2}}-\frac{\mu_{3} F_{2} e^{\rho_{5}}}{\left(e^{\rho_{2}}+a e^{\rho_{6}}\right)^{2}}, \\
& \delta_{2}=\frac{F^{2} e^{\rho_{2}}\left(\mu_{2} \alpha w+\mu_{1} h\right)}{\left(1+h F e^{\rho_{1}}+w F e^{\rho_{3}}\right)^{2}}-\frac{\mu_{1} F}{\left(1+h F e^{\rho_{2}}+w F e^{\rho_{4}}\right)^{2}}-\frac{\mu_{3} \beta b^{u} e^{\rho_{5}}}{\left(D+e^{\rho_{6}}\right)^{2}}-\frac{\mu_{3} \beta b^{u}}{D+e^{\rho_{6}}}, \\
& \delta_{3}=\frac{\mu_{3} F_{2} e^{\rho_{2}}}{\left(e^{\rho_{2}}+a e^{\rho_{6}}\right)^{2}}-\frac{\mu_{1} F_{1} e^{\rho_{1}}}{\left(e^{\rho_{1}}+a e^{\rho_{5}}\right)^{2}}-\frac{\mu_{2} b^{u} e^{\rho_{5}}}{\left(D+e^{\rho_{6}}\right)^{2}}-\frac{\mu_{2} b^{u}}{D+e^{\rho_{6}}}-\frac{\mu_{3} \beta b^{u} e^{\rho_{3}}}{\left(D+e^{\rho_{6}}\right)^{2}} .
\end{aligned}
$$

If the conditions of Theorem 6.1 hold, then $V(t)$ is monotonic decreasing on $[0, \infty)$. Now, integrating inequality (6.4) from 0 to $t$, we have

$V(t)+\delta_{1} \int_{0}^{t}|x(t)-\widetilde{x}(t)| d t+\delta_{2} \int_{0}^{t}|y(t)-\widetilde{y}(t)| d t+\delta_{3} \int_{0}^{t}|z(t)-\widetilde{z}(t)| d t \leq V(0)<\infty, \forall t \geq 0$.

Hence, by Lemma 5.1, we have

$$
\lim _{t \rightarrow \infty}|x(t)-\widetilde{x}(t)|=0, \lim _{t \rightarrow \infty}|y(t)-\widetilde{y}(t)|=0, \lim _{t \rightarrow \infty}|z(t)-\widetilde{z}(t)|=0 .
$$

Therefore, the positive periodic solution $(\widetilde{x}(t), \widetilde{y}(t), \widetilde{z}(t))$ is globally attractive.

To prove that the globally attractive periodic solution $(\widetilde{x}(t), \widetilde{y}(t), \widetilde{z}(t))$ is unique, we assume that $\left(\widetilde{x}_{1}(t), \widetilde{y}_{1}(t), \widetilde{z}_{1}(t)\right)$ is another globally attractive periodic solution of system (3.5) with period 1. If this solution is different from the solution $(\widetilde{x}(t), \widetilde{y}(t), \widetilde{z}(t))$, then there exists atleast one $\xi \in[0,1]$ such that $\widetilde{x}(\xi) \neq \widetilde{x}_{1}(\xi)$, which means $\left|\widetilde{x}(\xi)-\widetilde{x}_{1}(\xi)\right|=\epsilon_{11}>0$. Thus,

$$
\begin{aligned}
\epsilon_{11} & =\lim _{n \rightarrow \infty}\left|\widetilde{x}(\xi+n)-\widetilde{x}_{1}(\xi+n)\right| \\
& =\lim _{t \rightarrow \infty}\left|\widetilde{x}(t)-\widetilde{x}_{1}(t)\right|>0,
\end{aligned}
$$

which contradicts the fact that the periodic solution $(\widetilde{x}(t), \widetilde{y}(t), \widetilde{z}(t))$ is globally attractive. Therefore, $\widetilde{x}(t)=\widetilde{x}_{1}(t), \forall t \in[0,1]$. Similar arguments can be used for other components $\widetilde{y}(t)$ and $\widetilde{z}(t)$ also. Hence, the system (3.5) has unique positive 1-periodic solution, which is globally attractive.

\section{Existence of almost positive periodic solutions}

In a more general case, when we consider the effects of environmental factors, almost periodicity is sometimes more realistic and more general than periodicity because there is no priori reason to expect the existence of periodic solutions. We assume here that the predation and harvesting rates are almost periodic functions. We obtain sufficient conditions for the existence of a unique globally attractive positive almost periodic solution of system (3.5).

Consider almost periodic system

$$
x^{\prime}=f(t, x),
$$


where $f(t, x) \in C\left(\mathbb{R} \times \Gamma, \mathbb{R}^{n}\right), \Gamma=\{x:|x|<B\}$ and $f(t, x)$ is almost periodic in $t$ uniformly for $x \in \Gamma$. By using Lyapunov function, we discuss the existence of an almost periodic solution which is uniformly asymptotically stable in the whole region. For this, we consider the following system corresponding to system (7.1)

$$
x^{\prime}=f(t, x), y^{\prime}=f(t, y) .
$$

Lemma 7.1 Suppose that there exists a Lyapunov function $V(t, x, y)$ defined on $0 \leq t<\infty,|x|<B,|y|<B$, which satisfies the following conditions:

(1) $a(|x-y|) \leq V(t, x, y) \leq b(|x-y|)$, where $a(r)$ and $b(r)$ are continuous, increasing and positive definite.

(2) $\left|V\left(t, x_{1}, y_{1}\right)-V\left(t, x_{2}, y_{2}\right)\right| \leq k\left\{\left|x_{1}-x_{2}\right|+\left|y_{1}-y_{2}\right|\right\}$, where $k>0$ is a constant.

(3) $\dot{V}(t, x, y) \leq-\alpha V(t, x, y)$, where $\alpha>0$ is a constant.

Then in the region $\mathbb{R} \times \Gamma$, there exists a unique uniformly asymptotically stable almost periodic solution of system (7.1), which is bounded by B.

Let us denote $m_{i}^{\epsilon}=m_{i}$ and $M_{i}^{\epsilon}=M_{i}$ for $i=1,2,3$ in the region $\Gamma_{\epsilon}$. Assume that the conditions of Lemma 4.2 are satisfied, then system (5.2) is positively invariant and ultimately bounded in the region

$$
\begin{gathered}
\Gamma^{*}=\left\{(x, y, z) \in \mathbb{R}^{3} \mid \ln \left\{m_{1}\right\} \leq U(t) \leq \ln \left\{M_{1}\right\}, \ln \left\{m_{2}\right\} \leq V(t) \leq \ln \left\{M_{2}\right\},\right. \\
\left.\ln \left\{m_{3}\right\} \leq W(t) \leq \ln \left\{M_{3}\right\}\right\} .
\end{gathered}
$$

Let $S$ be the set of all solutions $(x(t), y(t), z(t))$ of system (3.5) satisfying $m_{1} \leq$ $x(t) \leq M_{1}, m_{2} \leq y(t) \leq M_{2}, m_{3} \leq z(t) \leq M_{3}, \forall t \in[0, \infty)$.

Lemma 7.2 The set $S$ is non empty.

Proof. Since $m_{1} \leq x_{0} \leq M_{1}, m_{2} \leq y_{0} \leq M_{2}, m_{3} \leq z_{0} \leq M_{3}$, then using Lemma 4.2, system (3.5) has solution $(x(t), y(t), z(t))$ satisfying $m_{1} \leq x(t) \leq M_{1}, m_{2} \leq$ $y(t) \leq M_{2}, m_{3} \leq z(t) \leq M_{3}, \forall t \in[0, \infty)$. Since $q(t)$ and $b(t)$ are almost periodic, there exists a sequence $\left\{t_{n}\right\}, t_{n} \rightarrow \infty$ as $n \rightarrow \infty$ such that $b\left(t+t_{n}\right) \rightarrow b(t)$ and $q\left(t+t_{n}\right) \rightarrow q(t)$ as $n \rightarrow \infty$ uniformly on $[0, \infty)$.

Now, we claim that $\left\{x\left(t+t_{n}\right)\right\},\left\{y\left(t+t_{n}\right)\right\}$ and $\left\{z\left(t+t_{n}\right)\right\}$ are uniformly bounded and equicontinuous on any bounded interval in $[0, \infty)$. Let $\left[\alpha_{1}, \beta_{1}\right] \subset[0, \infty)$ be a bounded interval and $\alpha_{1}+t_{n}>t_{0}$, then $t+t_{n}>t_{0}$ for any $t_{0} \in \mathbb{R}^{+}$and $t \in\left[\alpha_{1}, \beta_{1}\right]$. So, $m_{1} \leq x\left(t+t_{n}\right) \leq M_{1}, m_{2} \leq y\left(t+t_{n}\right) \leq M_{2}, m_{3} \leq z\left(t+t_{n}\right) \leq M_{3}, \forall t \in\left[\alpha_{1}, \beta_{1}\right]$. Hence, $\left\{x\left(t+t_{n}\right)\right\},\left\{y\left(t+t_{n}\right)\right\}$ and $\left\{z\left(t+t_{n}\right)\right\}$ are uniformly bounded. 
Now, using mean value theorem of differential calculus, we have

$$
\begin{gathered}
\left|x\left(t_{1}+t_{n}\right)-x\left(t_{2}+t_{n}\right)\right| \leq\left[r M_{1}\left(1+\frac{M_{1}}{K}\right)+\frac{F M_{1} M_{2}}{1+h F m_{1}+w F m_{2}}\right. \\
\left.+\frac{F_{1} M_{1} M_{3}}{m_{1}+a m_{3}}\right]\left|t_{1}-t_{2}\right|, \\
\left|y\left(t_{1}+t_{n}\right)-y\left(t_{2}+t_{n}\right)\right| \leq\left[\frac{\alpha F M_{1} M_{2}}{1+h F m_{1}+w F m_{2}}+\frac{b^{u} M_{2} M_{3}}{D+m_{3}}\right. \\
\left.+d_{1} M_{2}\right]\left|t_{1}-t_{2}\right|, \\
\left|z\left(t_{1}+t_{n}\right)-z\left(t_{2}+t_{n}\right)\right| \leq\left[\frac{\beta b^{u} M_{2} M_{3}}{D+m_{3}}+\frac{F_{2} M_{1} M_{3}}{m_{1}+a m_{2}}+d_{2} M_{3}\right. \\
\left.+q^{u} M_{3} E\right]\left|t_{1}-t_{2}\right| .
\end{gathered}
$$

In these inequalities, we see that $\left\{x\left(t+t_{n}\right)\right\},\left\{y\left(t+t_{n}\right)\right\}$ and $\left\{z\left(t+t_{n}\right)\right\}$ are equicontinuous on $\left[\alpha_{1}, \beta_{1}\right]$. Here, $\left[\alpha_{1}, \beta_{1}\right]$ is arbitrary. So, by Ascoli-Arzela theorem, there exists a subsequence of $\left\{t_{n_{k}}\right\}$ such that $x\left(t+t_{n_{k}}\right) \rightarrow p_{1}(t), y\left(t+t_{n_{k}}\right) \rightarrow p_{2}(t)$ and $z\left(t+t_{n_{k}}\right) \rightarrow p_{3}(t)$ as $n_{k} \rightarrow \infty$ uniformly in $t$ on any bounded interval in $[0, \infty)$. Furthermore,

$$
\begin{aligned}
& \frac{d x\left(t+t_{n_{k}}\right)}{d t}=r x\left(t+t_{n_{k}}\right)\left(1-\frac{x\left(t+t_{n_{k}}\right)}{K}\right)-\frac{F x\left(t+t_{n_{k}}\right) y\left(t+t_{n_{k}}\right)}{1+h F x\left(t+t_{n_{k}}\right)+w F y\left(t+t_{n_{k}}\right)} \\
& -\frac{F_{1} x\left(t+t_{n_{k}}\right) z\left(t+t_{n_{k}}\right)}{x\left(t+t_{n_{k}}\right)+a z\left(t+t_{n_{k}}\right)}, \\
& \frac{d y\left(t+t_{n_{k}}\right)}{d t}=\frac{\alpha F x\left(t+t_{n_{k}}\right) y\left(t+t_{n_{k}}\right)}{1+h F x\left(t+t_{n_{k}}\right)+w F y\left(t+t_{n_{k}}\right)}-\frac{b\left(t+t_{n_{k}}\right) y\left(t+t_{n_{k}}\right) z\left(t+t_{n_{k}}\right)}{D+z\left(t+t_{n_{k}}\right)} \\
& -d_{1} y\left(t+t_{n_{k}}\right) \text {, } \\
& \frac{d z\left(t+t_{n_{k}}\right)}{d t}=\frac{\beta b\left(t+t_{n_{k}}\right) y\left(t+t_{n_{k}}\right) z\left(t+t_{n_{k}}\right)}{D+z\left(t+t_{n_{k}}\right)}+\frac{F_{2} x\left(t+t_{n_{k}}\right) z\left(t+t_{n_{k}}\right)}{x\left(t+t_{n_{k}}\right)+a z\left(t+t_{n_{k}}\right)}-d_{2} z\left(t+t_{n_{k}}\right) \\
& -q\left(t+t_{n_{k}}\right) E z\left(t+t_{n_{k}}\right) .
\end{aligned}
$$

Let $n_{k} \rightarrow \infty$, then

$$
\begin{aligned}
& \frac{d p_{1}(t)}{d t}=r p_{1}(t)\left(1-\frac{p_{1}(t)}{K}\right)-\frac{F p_{1}(t) p_{2}(t)}{1+h F p_{1}(t)+w F p_{2}(t)}-\frac{F_{1} p_{1}(t) p_{3}(t)}{p_{1}(t)+a p_{3}(t)} \\
& \frac{d p_{2}(t)}{d t}=\frac{\alpha F p_{1}(t) p_{2}(t)}{1+h F p_{1}(t)+w F p_{2}(t)}-\frac{b(t) p_{2}(t) p_{3}(t)}{D+p_{3}(t)}-d_{1} p_{2}(t) \\
& \frac{d p_{3}(t)}{d t}=\frac{\beta b(t) p_{2}(t) p_{3}(t)}{D+p_{3}(t)}+\frac{F_{2} p_{1}(t) p_{3}(t)}{p_{1}(t)+a p_{3}(t)}-d_{2} p_{3}(t)-q(t) E p_{3}(t) .
\end{aligned}
$$

It is clear that $\left(p_{1}(t), p_{2}(t), p_{3}(t)\right)$ is a solution of the system (3.5) and $m_{1} \leq p_{1}(t) \leq$ $M_{1}, m_{2} \leq p_{2}(t) \leq M_{2}$ and $m_{3} \leq p_{3}(t) \leq M_{3}, \forall t \in[0, \infty)$. Hence, the proof is complete.

Theorem 7.1 Assume that the conditions of Lemma 4.2 are satisfied, then system (5.2) has a unique uniformly asymptotically stable almost periodic solution in $\Gamma^{*}$ 
provided the following conditions are satisfied:

$$
\begin{aligned}
& \frac{r}{K}-\frac{F\left\{\alpha+F M_{2}(\alpha w-h)\right\}}{\left(1+h F m_{1}+w F m_{2}\right)^{2}}-\frac{M_{3}\left(a F_{2}-F_{1}\right)}{\left(m_{1}+a m_{3}\right)^{2}}>0, \\
& \inf _{t \in \mathbb{R}}\left\{\frac{F\left\{m_{1}(\alpha w-h)-1\right\}}{\left(1+h F M_{1}+w F M_{2}\right)^{2}}-\frac{\beta b(t)\left(D+M_{3}\right)}{\left(D+M_{3}\right)^{2}}\right\}>0, \\
& \inf _{t \in R}\left\{\frac{m_{1}\left(a F_{2}-F_{1}\right)}{\left(M_{1}+a M_{3}\right)^{2}}+\frac{b(t)\left(\beta m_{2}-D\right)}{\left(D+M_{3}\right)^{2}}\right\}>0 .
\end{aligned}
$$

Proof. To prove that system (3.5) has unique uniformly asymptotically stable almost periodic solution in $\Gamma^{*}$, it suffices to show that the system (5.2) has unique uniformly asymptotically stable almost periodic solution in $\Gamma^{*}$.

Consider the product systems

$$
\begin{aligned}
\dot{U}_{1}(t) & =r-\frac{r e^{U_{1}(t)}}{K}-\frac{F e^{V_{1}(t)}}{1+h F e^{U_{1}(t)}+w F e^{V_{1}(t)}}-\frac{F_{1} e^{W_{1}(t)}}{e^{U_{1}(t)}+a e^{W_{1}(t)}}, \\
\dot{V}_{1}(t) & =\frac{\alpha F e^{U_{1}(t)}}{1+h F e^{U_{1}(t)}+w F e^{V_{1}(t)}}-\frac{b(t) e^{W_{1}(t)}}{D+e^{W_{1}(t)}}-d_{1}, \\
\dot{W}_{1}(t) & =\frac{\beta b(t) e^{V_{1}(t)}}{D+e^{W_{1}(t)}}+\frac{F_{2} e^{U_{1}(t)}}{e^{U_{1}(t)}+a e^{W_{1}(t)}}-d_{2}-q(t) E ; \\
\dot{U}_{2}(t) & =r-\frac{r e^{U_{2}(t)}}{K}-\frac{F e^{V_{2}(t)}}{1+h F e^{U_{2}(t)}+w F e^{V_{2}(t)}}-\frac{F_{1} e^{W_{2}(t)}}{e^{U_{2}(t)}+a e^{W_{2}(t)}}, \\
\dot{V}_{2}(t) & =\frac{\alpha F e^{U_{2}(t)}}{1+h F e^{U_{2}(t)}+w F e^{V_{2}(t)}}-\frac{b(t) e^{W_{2}(t)}}{D+e^{W_{2}(t)}}-d_{1}, \\
\dot{W}_{2}(t) & =\frac{\beta b(t) e^{V_{2}(t)}}{D+e^{W_{2}(t)}}+\frac{F_{2} e^{U_{2}(t)}}{e^{U_{2}(t)}+a e^{W_{2}(t)}}-d_{2}-q(t) E
\end{aligned}
$$

and the Lyapunov function,

$$
V\left(t, U_{1}, V_{1}, W_{1}, U_{2}, V_{2}, W_{2}\right)=\left|U_{1}(t)-U_{2}(t)\right|+\left|V_{1}(t)-V_{2}(t)\right|+\left|W_{1}(t)-W_{2}(t)\right| .
$$

Then, the condition 1 of Lemma 7.1 is satisfied when $a(r)=b(r)=r, r \geq 0$.

In addition,

$$
\begin{gathered}
\left|V\left(t, U_{1}, V_{1}, W_{1}, U_{2}, V_{2}, W_{2}\right)-V\left(t, U_{3}, V_{3}, W_{3}, U_{4}, V_{4}, W_{4}\right)\right| \\
=\left(\left|U_{1}(t)-U_{2}(t)\right|+\left|V_{1}(t)-V_{2}(t)\right|+\left|W_{1}(t)-W_{2}(t)\right|\right) \\
\quad-\left(\left|U_{3}(t)-U_{4}(t)\right|+\left|V_{3}(t)-V_{4}(t)\right|+\left|W_{3}(t)-W_{4}(t)\right|\right) \\
\leq\left(\left|U_{1}(t)-U_{3}(t)\right|+\left|V_{1}(t)-V_{3}(t)\right|+\left|W_{1}(t)-W_{3}(t)\right|\right) \\
\quad+\left(\left|U_{2}(t)-U_{4}(t)\right|+\left|V_{2}(t)-V_{4}(t)\right|+\left|W_{2}(t)-W_{4}(t)\right|\right) \\
\leq\left\|\left(U_{1}(t), V_{1}(t), W_{1}(t)\right)-\left(U_{3}(t), V_{3}(t), W_{3}(t)\right)\right\| \\
+||\left(U_{2}(t), V_{2}(t), W_{2}(t)\right)-\left(U_{4}(t), V_{4}(t), W_{4}(t)\right) \|,
\end{gathered}
$$

which satisfies condition 2 of Lemma 7.1. 
Let $\left(U_{i}, V_{i}, W_{i}\right)^{T}, i=1,2$ be any two solutions of system (5.2). Now, calculating the upper right derivative of $V(t)$ along the solutions of system (5.2), we get

$$
\begin{aligned}
D^{+} V(t)=\operatorname{sgn} & \left(U_{1}(t)-U_{2}(t)\right)\left[-\frac{r}{K}\left(e^{U_{1}(t)}-e^{U_{2}(t)}\right)\right. \\
& -\left(\frac{F e^{V_{1}(t)}}{1+h F e^{U_{1}(t)}+w F e^{V_{1}(t)}}-\frac{F e^{V_{2}(t)}}{1+h F e^{U_{2}(t)}+w F e^{V_{2}(t)}}\right) \\
& \left.-\left(\frac{F_{1} e^{W_{1}(t)}}{e^{U_{1}(t)}+a e^{W_{1}(t)}}-\frac{F_{1} e^{W_{2}(t)}}{e^{U_{2}(t)}+a e^{W_{2}(t)}}\right)\right] \\
+ & s g n\left(V_{1}(t)-V_{2}(t)\right)\left[\left(\frac{\alpha F e^{U_{1}(t)}}{1+h F e^{U_{1}(t)}+w F e^{V_{1}(t)}}-\frac{\alpha F e^{U_{2}(t)}}{1+h F e^{U_{2}(t)}+w F e^{V_{2}(t)}}\right)\right. \\
& \left.-\left(\frac{b(t) e^{W_{1}(t)}}{D+e^{W_{1}(t)}}-\frac{b(t) e^{W_{2}(t)}}{D+e^{W_{2}(t)}}\right)\right] \\
+ & \operatorname{sgn}\left(W_{1}(t)-W_{2}(t)\right)\left[\left(\frac{\beta b(t) e^{V_{1}(t)}}{D+e^{W_{1}(t)}}-\frac{\beta b(t) e^{V_{2}(t)}}{D+e^{W_{2}(t)}}\right)+\left(\frac{F_{2} e^{U_{1}(t)}}{e^{U_{1}(t)+a e^{W_{1}(t)}}}-\frac{F_{2} e^{U_{2}(t)}}{e^{U_{2}(t)+a e^{W_{2}(t)}}}\right)\right] .
\end{aligned}
$$

After rearranging the terms, we have

$$
\begin{aligned}
& D^{+} V(t) \leq-\frac{r}{K}\left|e^{U_{1}(t)}-e^{U_{2}(t)}\right|+\frac{F\left|e^{V_{1}(t)}-e^{V_{2}(t)}\right|}{\left(1+h F e^{U_{1}(t)}+w F e^{V_{1}(t)}\right)\left(1+h F e^{U_{2}(t)}+w F e^{V_{2}(t)}\right)} \\
& +\frac{h F^{2} e^{U_{2}(t)}\left|e^{V_{1}(t)}-e^{V_{2}(t)}\right|-h F^{2} e^{V_{2}(t)}\left|e^{U_{1}(t)}-e^{U_{2}(t)}\right|}{\left(1+h F e^{U_{1}(t)}+w F e^{V_{1}(t)}\right)\left(1+h F e^{U_{2}(t)}+w F e^{V_{2}(t)}\right)} \\
& +\frac{F_{1} e^{U_{2}(t)}\left|e^{W_{1}(t)}-e^{W_{2}(t)}\right|-F_{1} e^{W_{2}(t)}\left|e^{U_{1}(t)}-e^{U_{2}(t)}\right|}{\left(e^{U_{1}(t)}+a e^{W_{1}(t)}\right)\left(e^{U_{2}(t)}+a e^{W_{2}(t)}\right)} \\
& +\frac{\alpha F\left|e^{U_{1}(t)}-e^{U_{2}(t)}\right|}{\left(1+h F e^{U_{1}(t)}+w F e^{V_{1}(t)}\right)\left(1+h F e^{U_{2}(t)}+w F e^{V_{2}(t)}\right)} \\
& +\frac{\alpha w F^{2} e^{V_{2}(t)}\left|e^{U_{1}(t)}-e^{U_{2}(t)}\right|-\alpha w F^{2} e^{U_{2}(t)}\left|e^{V_{1}(t)}-e^{V_{2}(t)}\right|}{\left(1+h F e^{U_{1}(t)}+w F e^{V_{1}(t)}\right)\left(1+h F e^{U_{2}(t)}+w F e^{V_{2}(t)}\right)}+\frac{b(t) D\left|e^{W_{1}(t)}-e^{W_{2}(t)}\right|}{\left(D+e^{W_{1}(t)}\right)\left(D+e^{W_{2}(t)}\right)} \\
& +\frac{\beta b(t) D\left|e^{V_{1}(t)}-e^{V_{2}(t)}\right|}{\left(D+e^{W_{1}(t)}\right)\left(D+e^{W_{2}(t)}\right)}+\frac{\beta b(t) e^{W_{2}(t)}\left|e^{V_{1}(t)}-e^{V_{2}(t)}\right|-\beta b(t) e^{V_{2}(t)}\left|e^{W_{1}(t)}-e^{W_{2}(t)}\right|}{\left(D+e^{W_{1}(t)}\right)\left(D+e^{W_{2}(t)}\right)} \\
& +\frac{a F_{2} e^{W_{2}(t)}\left|e^{U_{1}(t)}-e^{U_{2}(t)}\right|-a F_{2} e^{U_{2}(t)}\left|e^{W_{1}(t)}-e^{W_{2}(t)}\right|}{\left(e^{U_{1}(t)}+a e^{W_{1}(t)}\right)\left(e^{U_{2}(t)}+a e^{W_{2}(t)}\right)} .
\end{aligned}
$$

We have,

$$
\begin{aligned}
D^{+} V(t) \leq- & \left|e^{U_{1}(t)}-e^{U_{2}(t)}\right|\left\{\frac{r}{K}-\frac{\alpha F+F^{2} M_{2}(\alpha w-h)}{\left(1+h F m_{1}+w F m_{2}\right)^{2}}-\frac{M_{3}\left(a F_{2}-F_{1}\right)}{\left(m_{1}+a m_{3}\right)^{2}}\right\} \\
& -\left|e^{V_{1}(t)}-e^{V_{2}(t)}\right|\left\{\frac{F\left\{m_{1} F(\alpha w-h)-1\right\}}{\left(1+h F M_{1}+w F M_{2}\right)^{2}}-\frac{\beta b(t)\left(D+M_{3}\right)}{\left(D+m_{3}\right)^{2}}\right\} \\
- & \left|e^{W_{1}(t)}-e^{W_{2}(t)}\right|\left\{\frac{m_{1}\left(a F_{2}-F_{1}\right)}{\left(M_{1}+a M_{3}\right)^{2}}+\frac{b(t)\left(\beta m_{2}-D\right)}{\left(D+M_{3}\right)^{2}}\right\} .
\end{aligned}
$$

Note that $U_{i}, V_{i}$ and $W_{i}$ are continuous functions on the bounded region $\Gamma^{*}$. 
Using mean value theorem, we have

$$
\begin{aligned}
\left|e^{U_{1}(t)}-e^{U_{2}(t)}\right| & =e^{\theta_{1}(t)}\left|U_{1}(t)-U_{2}(t)\right|, \\
\left|e^{V_{1}(t)}-e^{V_{2}(t)}\right| & =e^{\theta_{2}(t)}\left|V_{1}(t)-V_{2}(t)\right|, \\
\left|e^{W_{1}(t)}-e^{W_{2}(t)}\right| & =e^{\theta_{3}(t)}\left|W_{1}(t)-W_{2}(t)\right|,
\end{aligned}
$$

where

$$
U_{1}(t) \leq \theta_{1}(t) \leq U_{2}(t), V_{1}(t) \leq \theta_{2}(t) \leq V_{2}(t), W_{1}(t) \leq \theta_{3}(t) \leq W_{2}(t) .
$$

Thus, we have

$$
\begin{gathered}
D^{+} V(t) \leq-m_{1}\left|U_{1}(t)-U_{2}(t)\right|\left(\frac{r}{K}-\frac{\alpha F+F^{2} M_{2}(\alpha w-h)}{\left(1+h F m_{1}+w F m_{2}\right)^{2}}-\frac{M_{3}\left(a F_{2}-F_{1}\right)}{\left(m_{1}+a m_{3}\right)^{2}}\right) \\
-m_{2}\left|V_{1}(t)-V_{2}(t)\right|\left(\frac{F\left\{F m_{1}(\alpha w-h)-1\right\}}{\left(1+h F M_{1}+w F M_{2}\right)^{2}}-\frac{\beta b(t)\left(D+M_{3}\right)}{\left(D+m_{3}\right)^{2}}\right) \\
\quad-m_{3}\left|W_{1}(t)-W_{2}(t)\right|\left(\frac{b(t)\left(\beta m_{2}-D\right)}{\left(D+M_{3}\right)^{2}}+\frac{m_{1}\left(a F_{2}-F_{1}\right)}{\left(M_{1}+a M_{3}\right)^{2}}\right) \\
\leq-\mu\left(\left|U_{1}(t)-U_{2}(t)\right|+\left|V_{1}(t)-V_{2}(t)\right|+\left|W_{1}(t)-W_{2}(t)\right|\right) \\
\leq-\mu\left\|\left(U_{1}(t), V_{1}(t), W_{1}(t)\right)-\left(U_{2}(t), V_{2}(t), W_{2}(t)\right)\right\|,
\end{gathered}
$$

where

$$
\begin{aligned}
\mu=\min \{ & m_{1}\left(\frac{r}{K}-\frac{\alpha F+F^{2} M_{2}(\alpha w-h)}{\left(1+h F m_{1}+w F m_{2}\right)^{2}}-\frac{M_{3}\left(a F_{2}-F_{1}\right)}{\left(m_{1}+a m_{3}\right)^{2}}\right), \\
& \inf _{t \in \mathbb{R}} m_{2}\left(\frac{F\left\{F m_{1}(\alpha w-h)-1\right\}}{\left(1+h F M_{1}+w F M_{2}\right)^{2}}-\frac{\beta b(t)\left(D+M_{3}\right)}{\left(D+m_{3}\right)^{2}}\right), \\
& \left.\lim _{t \in \mathbb{R}} m_{3}\left(\frac{b(t)\left(\beta m_{2}-D\right)}{\left(D+M_{3}\right)^{2}}+\frac{m_{1}\left(a F_{2}-F_{1}\right)}{\left(M_{1}+a M_{3}\right)^{2}}\right)\right\}>0 .
\end{aligned}
$$

Thus, the condition 3 of Lemma 7.1 is verified. So, we conclude that the system (5.2) has unique uniformly asymptotically stable almost periodic solution in $\Gamma^{*}$. Hence, the system (3.5) has almost periodic solution. The proof is now complete. $\square$

\section{Numerical simulations}

Here, we perform the numerical simulations to investigate the dynamical behaviors of system (3.5) using the set of parameter values given in Table 1. Unless it is mentioned, the set of parameter values are the same as in Table 1 . We compare the dynamics of the nonautonomous system with the autonomous counterpart. Our aim is to explore different dynamical behaviors, including chaos. For the nonautonomous system (3.5), we consider that the rate parameters $b$ and $q$ are time dependent. More precisely, we consider that these biological parameters depends on water level. It is to be noted that the daily water level changes periodically throughout the year. Therefore, in the present investigation, we consider these parameters to be sinusoidal functions,

$$
b(t)=b+b_{11} \sin (\omega t), q(t)=q+q_{11} \sin (\omega t)
$$



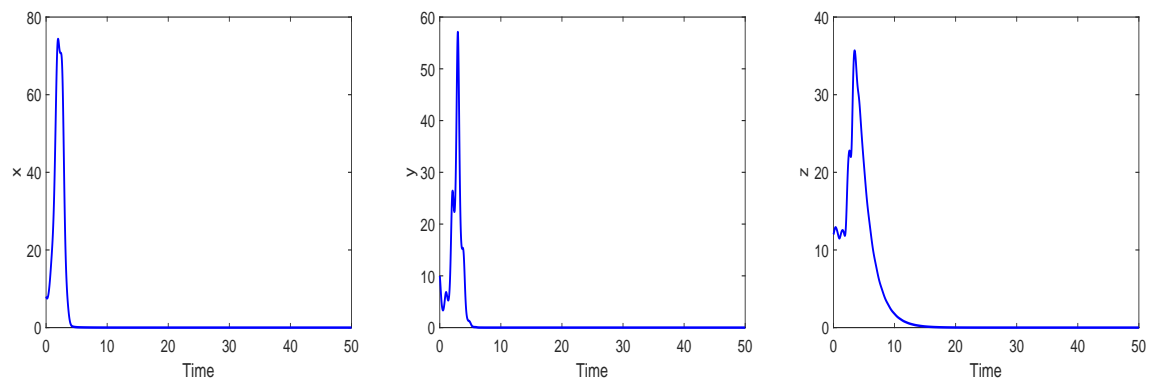

Fig. 2. System (3.5) shows extinction of $x$ population (first column), $y$ population (second column) and $z$ population (third column). Parameter values are the same as in Table 1 except $r=3.9$, $a=0.2, \alpha=0.59, F_{2}=0.2, b_{11}=2.1, q_{11}=0.9$.
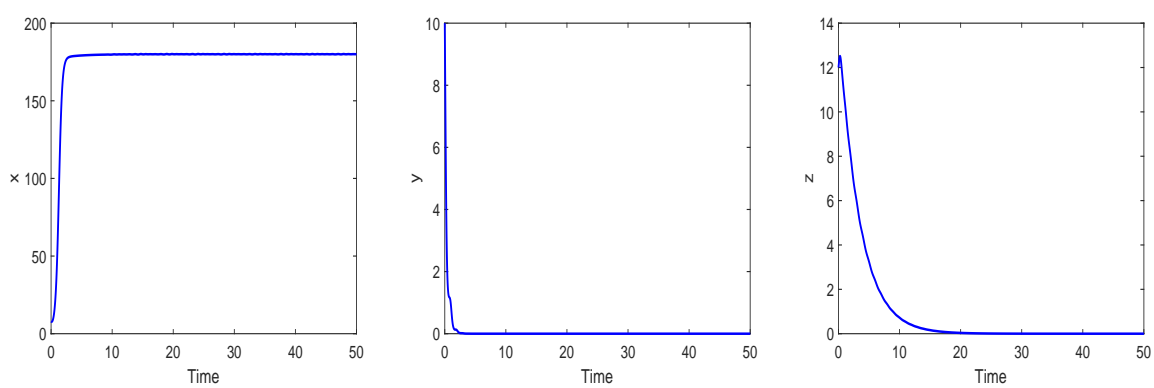

Fig. 3. System (3.5) shows extinction of $y$ population (second column) and $z$ population (third column). Parameter values are the same as in Table 1 except $r=3.9, a=0.2, \alpha=0.055, F_{2}=0.2$, $b_{11}=2.1, q_{11}=0.9$.

with period of 365 days. We have also considered these parameters in such a way that they are positive, continuous and bounded. Note that autonomous version of the system (3.5) can be obtained by assigning the seasonal forcing terms to be zero i.e., $b_{11}=q_{11}=0$.

We observe that the system (3.5) settles to population-free steady state at $r=3.9, a=0.2, \alpha=0.59, F_{2}=0.2, b_{11}=2.1, q_{11}=0.9$ (see Fig. 2) but on decreasing the value of $\alpha$ to 0.055 , the equilibrium with $x$ population only is achieved (see Fig. 3). Further, the system shows extinction of $z$ population at $\beta=0.001$, $F_{2}=0.05, E=0.7, b_{11}=0.1, q_{11}=0$ (see Fig. 4). Now, we plot the solution trajectories of the system (3.5) in the absence of seasonality, Fig. 5a. It is observed that the system is stable focus for the parameter values in Table 1. Next, we incorporate the effect of seasonality and see that there exists a positive periodic solution at $b_{11}=1$ and $q_{11}=0$ (see Fig. 5b). Therefore, the statement of Theorem 5.2 is verified. Next, we set $\alpha=0.55$, where the autonomous version of the system (3.5) shows limit cycle oscillation (see Fig. $6(\mathrm{a})$ ). We see that by setting $b_{11}=2.1$ 

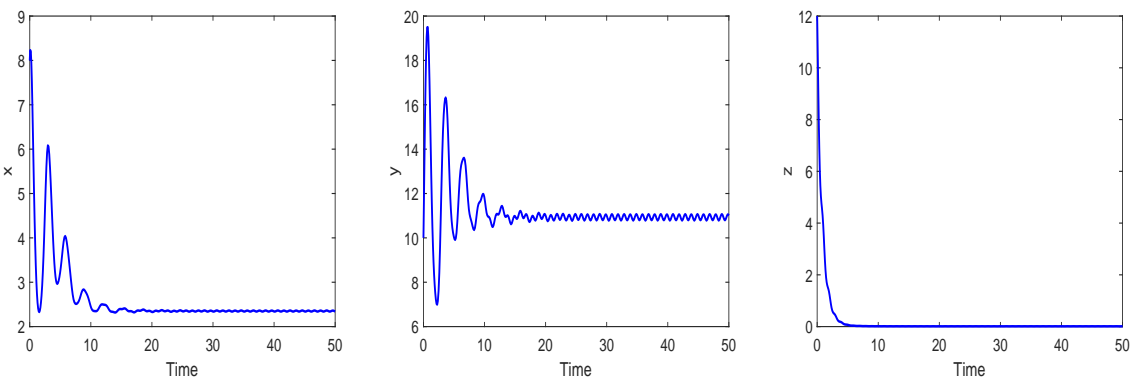

Fig. 4. System (3.5) shows extinction of $z$ population (third column). Parameter values are the same as in Table 1 except $\beta=0.001, F_{2}=0.05, E=0.7, b_{11}=0.1, q_{11}=0$.
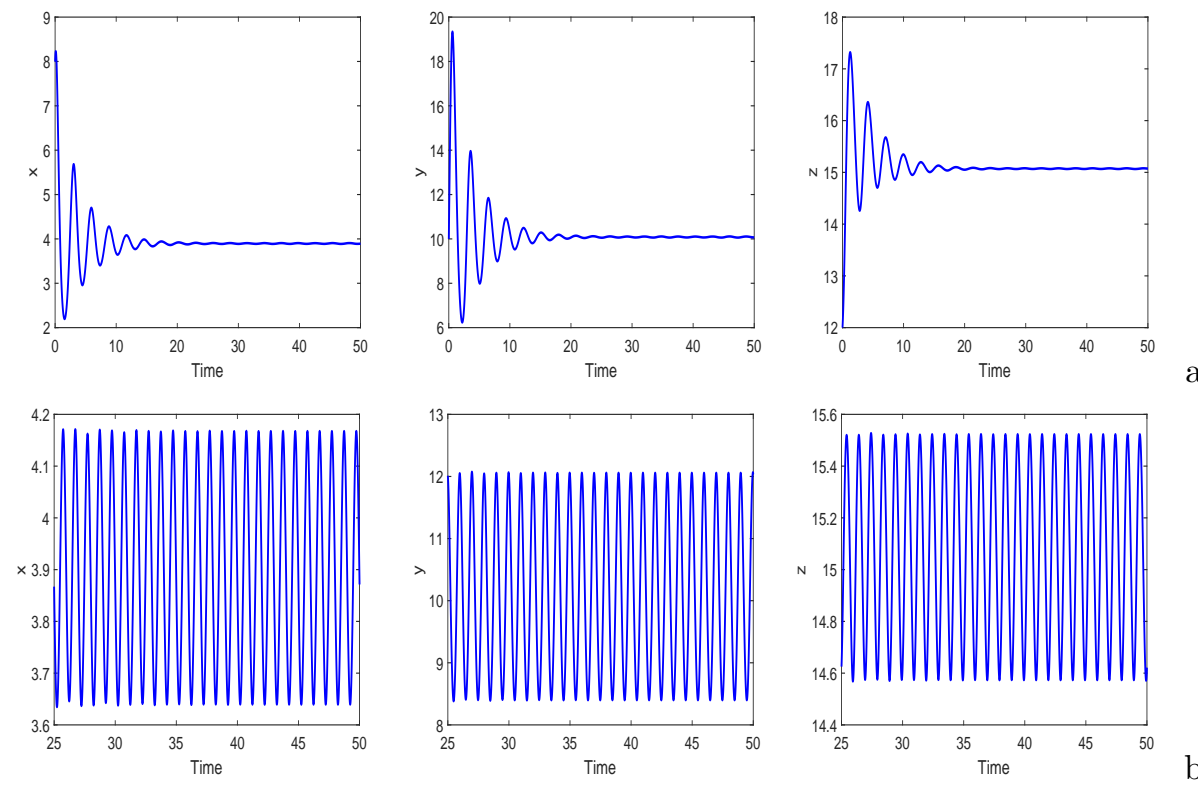

Fig. 5. System (3.5) exhibits (a) stable focus at $b_{11}=q_{11}=0$, and (b) periodic solution at $b_{11}=1$ and $q_{11}=0$. Rest of the parameters are at the same values as in Table 1 .

and $q_{11}=0$, the nonautonomous system (3.5) exhibits chaotic dynamics (see Fig. $6(\mathrm{~b}))$. The occurrence of chaotic oscillation may be explained through incommensurate limit cycles ${ }^{44,45}$. Thus, for the same set of the parameter values the nonautonomous system (3.5) exhibits positive periodic solution while the corresponding autonomous system shows stable focus. Further, the nonautonomous system (3.5) shows chaotic behavior if the corresponding autonomous system exhibits limit cycle oscillations. Further, we show global stability of the positive periodic solution of the nonautonomous system (3.5). We fix $b_{11}=1$ and $q_{11}=0$, and plot the solution 

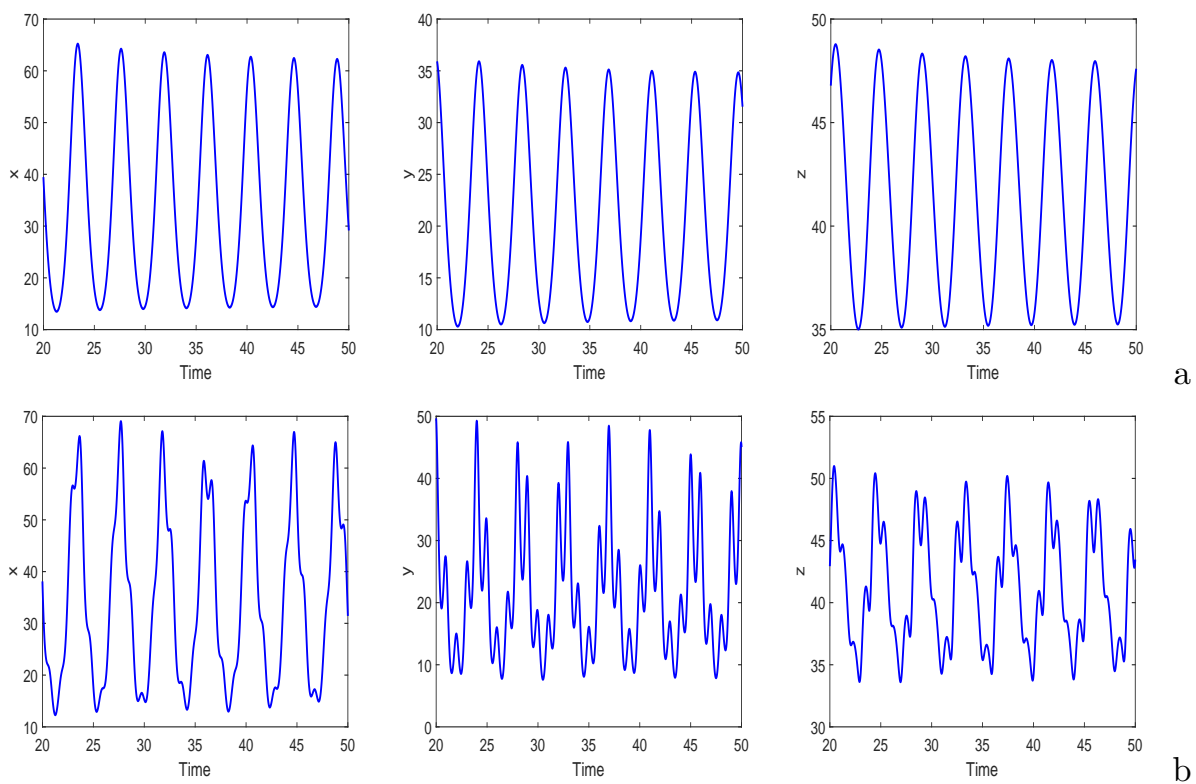

Fig. 6. System (3.5) exhibits (a) limit cycle oscillations at $\alpha=0.55, b_{11}=0, q_{11}=0$, and (b) chaotic dynamics at $\alpha=0.55, b_{11}=2.1, q_{11}=0$. Rest of the parameters are at the same values as in Table 1.
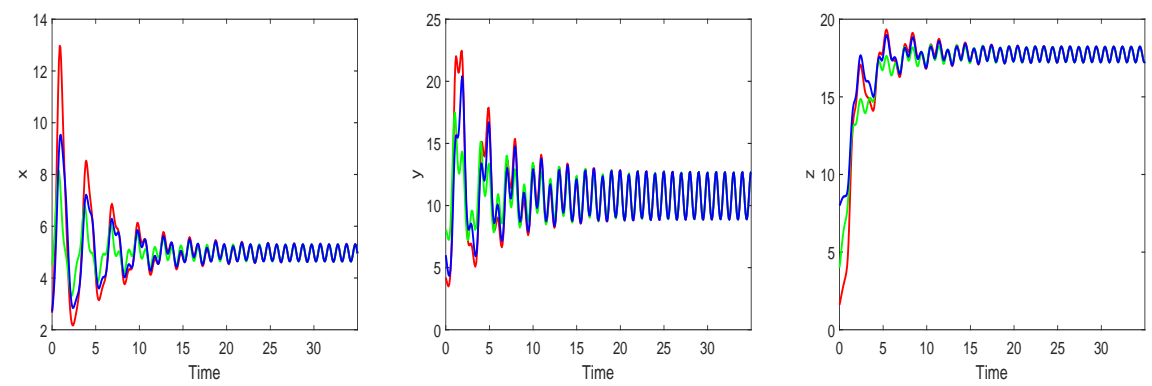

Fig. 7. Global stability of positive periodic solution for the system (3.5) at $b_{11}=1, q_{11}=0$, when other parameter values are same as in Table 1. Figure shows that solution trajectories starting from three different initial points $(2.65,4.2,1.6),(4.5,8,4)$ and $(2.7,6,8)$ ultimately converge to a unique positive periodic solution.

trajectories initiated from three different initial values, Fig. 7. It is apparent from the figure that all the periodic solutions initiating from three different initial values $(2.65,4.2,1.6),(4.5,8,4)$ and $(2.7,6,8)$ converge to a single periodic solution i.e., the positive periodic solution is globally asymptotically stable. Therefore, Theorem 6.1 for the global asymptotic stability of the positive periodic solution of the nonautonomous system (3.5) is verified. Now we set $r=3.9, w=0.067, \alpha=2, b_{11}=2.1$, 

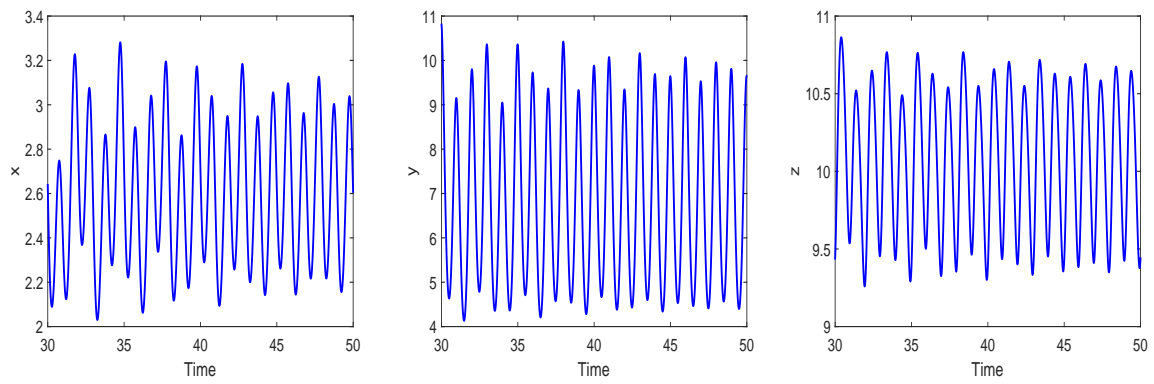

Fig. 8. System (3.5) exhibits almost periodic solution for $r=3.9, w=0.067, \alpha=2, b_{11}=2.1$, $q_{11}=0.9$. Rest of the parameters are at the same values as in Table 1 .
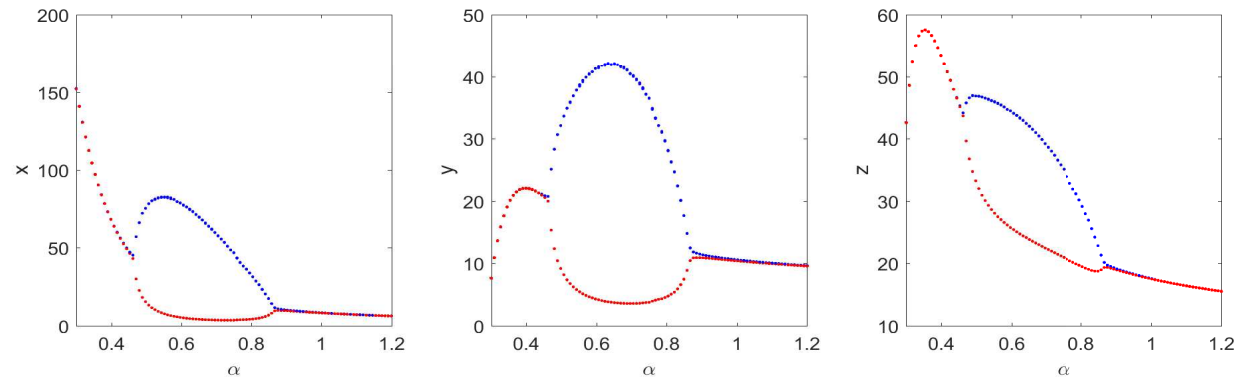

Fig. 9. Bifurcation diagram of system (3.5) with respect to $\alpha$ in the absence of seasonality i.e., $b_{11}=q_{11}=0$. Rest of the parameters are at the same values as in Table 1 . Here, the maximum and minimum values of the oscillations are plotted in blue and red colors, respectively.
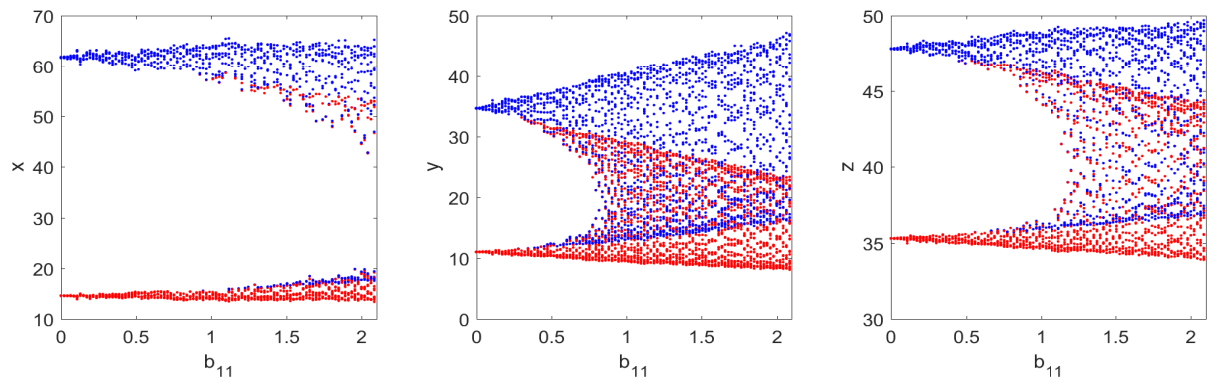

Fig. 10. Bifurcation diagram of system (3.5) with respect to $b_{11}$ for $\alpha=0.55$ and $q_{11}=0$. Rest of the parameters are at the same values as in Table 1 . Here, the maximum and minimum values of the oscillations are plotted in blue and red colors, respectively.

$q_{11}=0.9$, and see that the system (3.5) exhibits almost periodic solution, Fig. 8 .

Thus, the statement of Theorem 7.1 is also verified. 

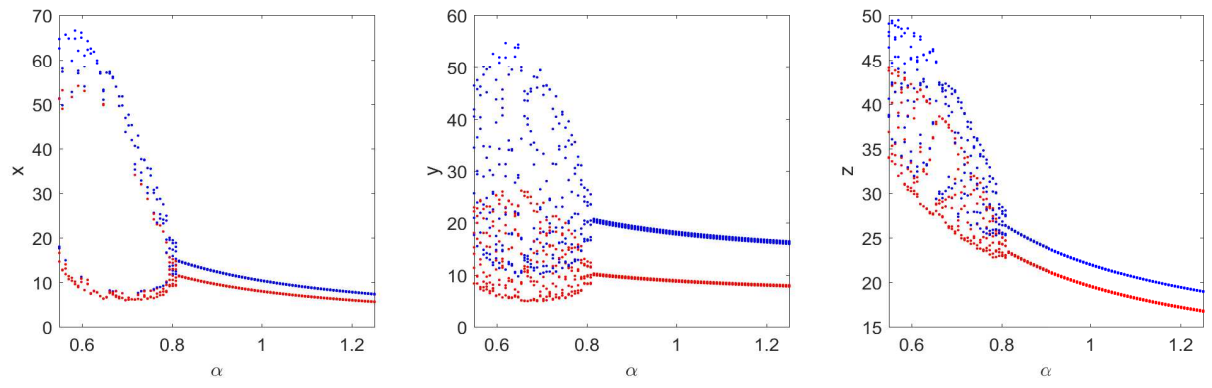

Fig. 11. Bifurcation diagram of system (3.5) with respect to $\alpha$ for $b_{11}=2.1$ and $q_{11}=0$. Rest of the parameters are at the same values as in Table 1. Here, the maximum and minimum values of the oscillations are plotted in blue and red colors, respectively.

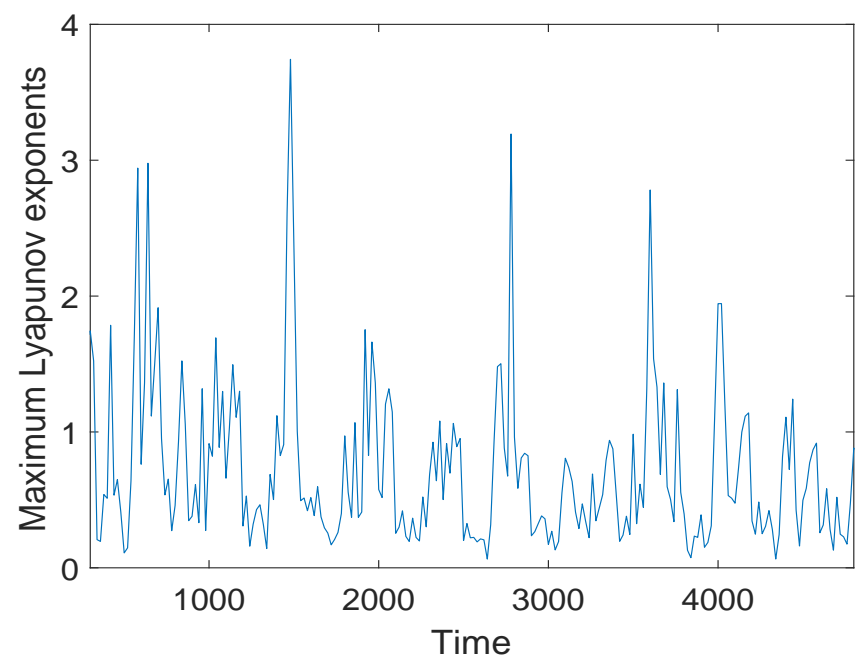

Fig. 12. Figure shows the maximum Lyapunov exponent of the system (3.5) for $\alpha=0.55, b_{11}=2.1$, $q_{11}=0$. Rest of the parameters are at the same values as in Table 1. In the figure, positive values of the maximum Lyapunov exponent confirms the occurrence of chaotic oscillation.

Next, we draw the bifurcation diagram of autonomous version of the system (3.5) by varying the parameter $\alpha$, Fig. 9 . We see that the system is stable for very low and very high values of $\alpha$, and undergoes first supercritical and then subcritical Hopf bifurcations on increasing the values of $\alpha$. Now, we fix the value of $\alpha$ at 0.55 , where the autonomous version of the system (3.5) shows limit cycle oscillations. To visualize the effect of the parameter $b_{11}$, we draw bifurcation diagram of the system (3.5) by varying $b_{11}$, Fig. 10. It is apparent from the figure that the nonautonomous system undergoes chaotic regime through quasi-periodic oscillations. However, the chaotic dynamics can be controlled and the system (3.5) returns to periodic oscilla- 
tions on increasing the values of $\alpha$, Fig. 11. Using the algorithm of ${ }^{46,47}$, we draw the maximum Lyapunov exponent of the system (3.5) to confirm its chaotic behavior reported in Fig. 10. The maximum Lyapunov exponent has proved to be the most useful dynamical diagnostic for chaotic systems, which is the average exponential rate of divergence or convergence of nearby orbits in phase space. The general idea of calculating the maximum Lyapunov exponent is to follow two nearby orbits and to calculate their average logarithmic rate of separation. Whenever they get too far apart, one of the orbits has to be moved back to the vicinity of the other along the line of separation. For a chaotic attractor, the maximum Lyapunov exponent is positive; for a bifurcation point, the maximum Lyapunov exponent is zero; if the maximum Lyapunov exponent is negative, this is corresponding to a fixed point or a periodic attractor. The maximum Lyapunov exponent corresponding to Fig. 10 have been computed and plotted in Fig. 12. We see that at $b_{11}=2.1$, the maximum Lyapunov exponent is positive, which denote that the system is chaotic.

\section{Conclusion}

Aquatic ecosystem is often altered by human activities. Water-level fluctuations are among the major driving forces for shallow lake ecosystem. This study provides results of the evolution of the ecosystem based on water management of the lake. We considered three species system, namely invertebrates, prey (small fish) and predator (large fish) in intention to study the impact of water level on persistence of fish populations. We also considered a time dependent harvesting of large fish to maintain ecological balance. It is to be noted that the level of water depends on temperature and rainfall. Therefore, such parameter is time dependent and its value follow the periodic (sinusoidal) function with lower bound greater than zero 48. A lot of studies showed the occurrence of limit cycle oscillations through a Hopfbifurcation by varying the water level fluctuation $33,34,49,50$. However, none of the previous studies conducted for the effect of water level fluctuation showed chaotic dynamics. In the present investigation, our aim is to extensively study the effect of water level fluctuation and explore how the system produces chaotic behavior due to presence of seasonality. Our analytical findings show that according to the values of the parameters, one can make suitable predictions about the asymptotic behavior of the overall three species system including permanence, and extinction of the species. The existence of periodic solutions has been explored, which should be viewed as a condition allowing for the survival of the species under consideration. The conditions for global stability of the unique positive periodic solution are also derived. Moreover, we obtained conditions for existence, uniqueness and stability of a positive almost periodic solution.

The analytical findings are well supported by numerical simulations. We consider periodic function (sinusoidal function) with a period of one year to incorporate the seasonal patterns of water level and harvesting rate of large fish. We have also studied the dynamical behavior of the system (3.5) by assuming these rate parameters 
to be independent of time. In the absence of seasonality, system (3.5) shows stable focus, whereas the nonautonomous system (3.5) shows a unique positive periodic solution with a period of one year. Further, we seen that if the autonomous system undergoes a Hopf-bifurcation, the corresponding nonautonomous system showed chaotic dynamics. We have observed that the assimilation fraction of prey and seasonal forcing have the synergism effect for producing chaotic oscillations. We also showed that for a range of the water level fluctuation, the positive periodic solution is globally asymptotically stable. Our nonautonomous system produces almost periodic solution for a particular set of parameter values. Moreover, the assimilation fraction of prey has capability to control chaos in the system. Our results evoke that the water level has an important effect on persistence of the species. Ecologically, if the water-levels are between critical values, then the two species can coexist and tend to fluctuate with the same period as the environment. On the contrary, at high levels of water, there are weak interactions between species and then the predator species goes to extinction. It is concluded that using water volume as control, it is possible to keep the levels of the populations at a required state.

\section{Acknowledgement}

Authors are grateful to Prof. Guido Badino, DBIOS, University of Turin, Italy for reviewing the ecological aspects of the paper. The research work of Abhijit Sarkar is supported by University Grants Commision, Government of India, New Delhi in the form of Senior Research Fellowship (No. F.16-6(DEC. 2016)/2017(NET)). Pankaj Kumar Tiwari is thankful to University Grants Commissions, New Delhi, India for providing financial support in form of Dr. D.S. Kothari post-doctoral fellowship (No.F.4-2/2006 (BSR)/MA/17-18/0021).

\section{References}

1. Kolding J, van Zwieten PAM, Relative lake level fluctuations and their influence on productivity and resilience in tropical lakes and reservoirs, Fish Res 115:99-109, 2012.

2. Piano E, Falasco E, Bona F, Mediterranean rivers: consequences of water scarcity on benthic algal chlorophyll-a content, J Limnol 76:39-48, 2016.

3. Scheffer $\mathrm{M}$ et al., Seasonality and chaos in a plankton-fish model, Theor Pop Biol 43:159-183, 1993.

4. Jul-Larsen E et al., Management, co-management or no management? Major dilemmas in southern African freshwater fisheries. 2. Case studies. FAO Fisheries Technical Paper, No. 426/2. Food \& Agriculture Organization of the United Nations, Rome 271, 2003.

5. Kolding J, van Zwieten PAM, Improving productivity in tropical lakes and reservoirs, Challenge Program on Water and Food, Aquatic Ecosystems and Fisheries Review Series I, WorldFish Center, Cairo 139, 2006.

6. Gownaris NJ et al., Fisheries and water level fluctuations in the world's largest desert lake, Ecohydrology 10:e1769, 2017.

7. Paukert CP et al., Designing a global assessment of climate change on inland fishes and fisheries: knowns and needs, Rev Fish Biol Fish 27:393-409, 2017. 
8. Wantzen KM, Junk WJ, Rothhaupt K-O, An extension of the floodpulse concept (FPC) for lakes, Hydrobiologia 613:151-170, 2008.

9. Zohary T, Ostrovsky I, Ecological impacts of excessive water level fluctuations in stratified freshwater lakes, Inland Waters 1:47-59, 2011.

10. Junk WJ, Bayley PB, Sparks RE, The flood pulse concept in river-floodplain systems, Can Spec Publ Fish Aquat Sci 106:110-127, 1989.

11. Doll P, Fiedler K, Zhang J, Global-scale analysis of river flow alterations due to water withdrawals and reservoirs, Hydrol Earth Syst Sci 13:2413-2432, 2009.

12. Haddeland I et al., Global water resources affected by human interventions and climate change, Proc Natl Acad Sci USA, 111:3251-3256, 2014.

13. Hill NM, Keddy PA, Wisheu IC, A hydrological model for predicting the effects of dams on the shoreline vegetation of lakes and reservoirs, Environ Manag 22:723-736, 1998.

14. Evtimova VV, Donohue I, Water-level fluctuations regulate the structure and functioning of natural lakes, Freshw Biol 61:251-264, 2016.

15. Kolding J, Population dynamics and life-history styles of Nile tilapia, Oreochromis niloticus, in Ferguson's Gulf, Lake Turkana, Kenya, Environ Biol Fish 37:25-46, 1993.

16. Gasith A, Gafny S, Effects of water level fluctuation on the structure and function of the littoral zone, In: Tilzer MM, Serruya C (Eds.), Large Lakes, Ecological Structure and Function, Springer, Berlin 156-171, 1990.

17. Coops H, Hosper SH, Water-level management as a tool for the restoration of shallow lakes in the Netherlands, Lake Reserv Manage 18(4):293-298, 2002.

18. Coops H, Beklioglu M, Crisman TL, The role of water-level fluctuations in shallow lake ecosystems-workshop conclusions, Hydrobiologia 506-509:23-27, 2003.

19. Kahl U, The impact of water level fuctuations on the year class strength of roach: Implications for fish stock management, Limnologica 38:258-268, 2008.

20. Wlosinski JH, Koljord ER, Effects of water levels on ecosystems, an annotated bibliography, Long Term Resource Monitoring Program, Technical Report 96-T007, 1996.

21. Fischer P, Ohl U, Effects of water-level fluctuations on the littoral benthic fish community in lakes: a mesocosm experiment, Behav Ecol 16(4):741-746, 2005.

22. Gownaris NJ et al., Water level fluctuations and the ecosystem functioning of lakes, J Great Lakes Res 44:1154-1163, 2018.

23. Yin XA, Yang ZF, A method to assess the alteration of water-level-fluctuation patterns in lakes, Procedia Environ Sci 13:2427-2436, 2012.

24. Lowe-McConnell RH, Ecological studies in tropical fish communities, Cambridge University Press, Cambridge 400, 1987.

25. Roy R, Tissot L, Argillier C, Environmental drivers of fish spatial distribution and activity in a reservoir with water level fluctuations, Hydrocol Appl 2018.

26. Mosepele K, Kolding J, Bokhutlo T, Fish community dynamics in an inland floodplain system of the Okavango Delta, Botswana, Ecohydrol Hydrobiol 17:89-102, 2017.

27. Lotka AJ, The elements of physical biology, Williams and Wilkins: Baltimore, 1925.

28. Volterra V, Variazioni e fluttuazioni del numero di individui in specie animali conviventi, Mem Accd Lincei 2:31-113, 1926.

29. Benzerdjeb S, Bouguima SM, Influence of water level in a prey-predator interaction, Nonlinear Studies 22(2):281-291, 2015.

30. Moussaoui A, Aziz-Alaoui MA, Bassaid S, Predator-prey dynamics with seasonal water-level fluctuations, J Biol Syst 26(04):495-510, 2018.

31. Moussaoui A, Bouguima SM, Seasonal influences on a prey-predator model, J Appl Math Comp 50(1-2):39-57, 2016.

32. Chiboub FN, Bouguima SM, Moussaoui A, The effect of water level in a prey-predator 
interactions: a nonlinear analysis study, Chaos Solit Fract 45:205-212, 2012.

33. Fellah NC, Bouguima SM, Moussaoui A, The effect of water level in a prey-predator interactions: A nonlinear analysis study, Chaos Solit Fract 45:205-212, 2012.

34. Moussaoui A, Bouguima SM, A prey-predator interaction under fluctuating level water, Math Meth Appl Sci 38:123-137, 2014.

35. DeAngelis DL et al., Fish population dynamics in a seasonally varying wetland, Ecol Model 221:1131-1137, 2010.

36. Menouer MA, Moussaoui A, Effects of consecutive water level fluctuations and harvesting on predator-prey interactions, Chaos Solit Fract 91:434-442, 2016.

37. Gaines RE, Mawhin JL, Coincidence degree and nonlinear differential equations, Springer-Verlag: Berlin, 1977.

38. Maiti SAP, Samanta GP, Rich dynamics of a food chain model with Hassell-Varley type functional responses, Appl Math Com 208:303-317, 2009.

39. Yoshizawa T, Stability theory and the existence of periodic solutions and almost periodic solutions, Berlin: Springer-Verlag New York Heidelberg, 1975.

40. Amerio L, Prouse G, Almost periodic functions and functional equations, New York: Springer Science+Business Media, 1971.

41. Ton TV, Hieu NT, Dynamics of species in a model with two predators and one prey, Nonlinear Anal 74:4868-4881, 2011.

42. Wang Q, Fan M, Wang K, Dynamics of a class of nonautonomous semi-ratio-dependent predator-prey system with functional responses, J Math Anal Appl 278:443-471, 2003.

43. Zhijun Z, Dynamics of a non-autonomous ratio-dependent food chain model, Appl Math Comp 215:1274-1287, 2009.

44. Hastings A, Powell T, Chaos in a three-species food chain, Ecology 72(3):896-903, 1991.

45. Guckenheimer J, Holmes P, Nonlinear oscillations, dynamical systems, and bifurcations of vector fields, Springer Science \& Business Media 42, 2013.

46. Park T, A matlab version of the lyapunov exponent estimation algorithm of wolf et al. - physica16d, 1985, https://www.mathworks.com/matlabcentral/fileexchange/ 48084-lyapunov-exponent-estimation-from-a-time-series-documentation-added.

47. Wolf A et al., Determining lyapunov exponents from a time series, Phys D 16(3):285$317,1985$.

48. Samanta $\mathrm{S}$ et al., Chaos in a nonautonomous eco-epidemiological model with delay, Appl Math Model 79:865-880, 2020.

49. Moussaoui A et al., The impact of water level fluctuations on a delayed prey-predator model, Nonlinear Anal RWA 21:170-184, 2015.

50. Moussaoui A, A reaction-diffusion equations modelling the effect of fluctuating water levels on prey-predator interactions, Appl Math Comput 268:1110-1121, 2015. 